MATHEMATICS OF COMPUTATION

Volume 74, Number 250, Pages 819-839

S 0025-5718(04)01712-0

Article electronically published on October 27, 2004

\title{
SUBDIVISION SCHEMES WITH NONNEGATIVE MASKS
}

\author{
XINLONG ZHOU
}

\begin{abstract}
The conjecture concerning the characterization of a convergent univariate subdivision algorithm with nonnegative finite mask is confirmed.
\end{abstract}

\section{INTRODUCTION}

Let $\mathbb{Z}$ denote the integer lattice. A univariate subdivision algorithm with a finitely supported mask $a=\left\{a_{j}\right\}_{j \in \mathbb{Z}}$ is given as follows: beginning with an initial sequence of data $v^{0}=\left\{v_{i}^{0}\right\}$, we set recursively new sequences of values $v^{k}$, by applying the rule

$$
v_{i}^{k}=\sum_{j \in \mathbb{Z}} a_{i-2 j} v_{j}^{k-1} .
$$

This algorithm is said to converge if for each finite $v^{0}=\left\{v_{i}^{0}\right\}$ there exists a continuous function $f_{v}$ such that $f_{v} \not \equiv 0$ for at least one $v^{0}$ and

$$
\lim _{k \rightarrow \infty} \sup _{i \in \mathbb{Z}}\left|f_{v}\left(\frac{i}{2^{k}}\right)-v_{i}^{k}\right|=0 .
$$

Obviously, the subdivision algorithm with mask $a=\left\{a_{j}\right\}_{j \in \mathbb{Z}}$ converges if and only if the polygon $f_{k}$ defined by the control points $\left(i / 2^{k}, v_{i}^{k}\right)^{T}$ converges uniformly to $f_{v}$. One can easily check

$$
f_{k}(x)=\sum_{i} v_{i}^{0} \sum_{j} a_{j}^{k} h\left(2^{k}(x-i)-j\right),
$$

where $h$ is the hat function and $a_{i}^{k}=\sum_{j} a_{j}^{k-1} a_{i-2 j}$ with $a_{j}^{1}=a_{j}$. Thus (1.1) for all such $v^{0}$ is equivalent to the uniform convergence of

$$
\sum_{j} a_{j}^{k} h\left(2^{k} x-j\right)
$$

On the other hand, define $S$ to be the operator given by

$$
S f(x)=\sum_{j} a_{j} f(2 x-j) .
$$

One gets

$$
S^{k} f(x)=\sum_{j} a_{j}^{k} f\left(2^{k} x-j\right), \quad k=1,2, \ldots
$$

Received by the editor December 13, 2002 and, in revised form, January 15, 2004.

2000 Mathematics Subject Classification. Primary 65D17, 26A15, 26A18, 39A10, 39B12.

Key words and phrases. Cascade algorithm, joint spectral radius, nonnegative mask, subdivision scheme.

(C)2004 American Mathematical Society 
The convergence of (1.1) is therefore equivalent to the uniform convergence of $S^{k} h$, which leads to the following cascade algorithm: let $g_{0}(x)=h(x)$; one defines recursively $g_{k}(x)$ by

$$
g_{k}(x)=\sum_{j} a_{j} g_{k-1}(2 x-j) .
$$

The limit $g=\lim _{k \rightarrow \infty} g_{k}=\lim _{k \rightarrow \infty} S^{k} h$ satisfies $S g=g$, i.e.,

$$
g(x)=\sum_{j \in \mathbb{Z}} a_{j} g(2 x-j) .
$$

This so-called two-scale delation equation and the above presented algorithms play an important role in wavelet analysis as well as in computer aided geometry design. A comprehensive discussion of this subject can be found in [2, 5, 10.

In order to characterize the convergence of the above algorithms, one uses the concept of joint spectral radius (see [11]). The joint spectral radius for two square matrices $A_{0}$ and $A_{1}$ is defined by

$$
\rho\left(A_{0}, A_{1}\right)=\lim _{n \rightarrow \infty} \sup _{k \leq n}\left\|A_{\epsilon_{1}} \cdots A_{\epsilon_{k}}\right\|^{\frac{1}{k}},
$$

where $\|\cdot\|$ is a given matrix-norm and $\epsilon_{l} \in\{0,1\}$. Thus, write $a(z)=\sum_{j} a_{j} z^{j}$ and $a(z)=(1+z) b(z)$ if $a(-1)=0$. The subdivision algorithm with finite mask $a=\left\{a_{j}\right\}_{j \in \mathbb{Z}}$ converges if and only if (see [2, 5, 10])

(i) $a(1)=2$ and $a(-1)=0$,

(ii) $\rho\left(B_{0}, B_{1}\right)<1$ where $B_{0}=\left[b_{2 j-i}\right]_{i, j}, B_{1}=\left[b_{2 j-i+1}\right]_{i, j}$ and $\left\{b_{l}\right\}$ is the coefficients of $b$.

Unfortunately, the determination of the joint spectral radius is generally NPhard by a result of Tsitsiklis and Blondel (see [13]). Thus, it seems difficult to determinate whether the considered spectral radius is less than one. Some partial results concerning the calculation of the joint spectral radius can be found in [1, 4, 7) 16] and the papers cited therein.

In this paper we focus on subdivision algorithms with nonnegative finite masks, a property possessed by many applications in geometric modelling (see, e.g., [3, 12]). A remarkable fact of this class is that the convergence does not depend on the actual values of the mask but rather on the support of the mask $I=\left\{i: a_{i}>0\right\}$ (see [10, 8]). Consequently the question was raised in [2] (see p. 55 of [2]) of identifying those $I$ such that given any nonnegative mask supported on $I$, the corresponding subdivision algorithm converges. By applying a suitable translation, we may always assume in the following discussion that a nonnegative mask $a=\left\{a_{j}\right\}$ has the form $a=\left\{a_{0}, \ldots, a_{N}\right\}$ with $a_{0}, a_{N}>0$. We believe that the answer to this question is (see [9]):

Conjecture. The subdivision algorithm associated with the nonnegative mask $a=$ $\left\{a_{0}, \ldots, a_{N}\right\}$ converges if and only if the following both hold:

(i) $a(1)=2, a(-1)=0$ and $0<a_{0}, a_{N}<1$,

(ii) the greatest common divisor of $\left\{j: a_{j}>0\right\}$ is 1 .

This conjecture is still not verified. On the other hand, it has been shown that the conditions are necessary (see 2, 9, 14]). There are various partial results which support this conjecture. Denote $S(a)=\left\{j: a_{j}>0\right\}$. Thus, Micchelli and Prautzsch (see [10]) prove that if (ii) is replaced by $S(a)=\{0,1, \ldots, N\}$, convergence follows. 
Gonsor (see [6]) shows that $S(a)=\{0,1, \ldots, N\}$ can be weakened by $\{0,1, N-$ $1, N\} \subseteq S(a)$, while Melkman (see 9]), among others, proves that if instead of (ii) it holds that $S(a) \supseteq\{0, p, q, p+q\}$ for $\operatorname{gcd}(p, q)=1$ or that $S(a)$ contains two successive integers, then convergence follows. Recently, Wang proves this conjecture for a class of masks. One of the main results in [15] is the following

Theorem 1.1. The subdivision algorithm with the nonnegative mask $a=\left\{a_{j}\right\}$ converges if instead of (ii) it holds that $\{r, p, q\} \subseteq S(a)$ such that $\operatorname{gcd}(p-r, q-r)=1$ and $q-r$ is even.

Improving the technique introduced in [15], we can now confirm this conjecture. In next section we first collect some lemmas from [15] and prove the conjecture by using a lemma (see Lemma 2.4), which will be verified in Section 3. The proof of Lemma 2.4 is the kernel of this paper.

\section{Proof of the conjecture}

Let us collect some results from [15. For this goal we should denote the vector $x$ in $\mathbb{R}^{N}$ by $x=\left(x_{0}, \ldots, x_{N-1}\right)^{T}$. For any $T \subset \mathbb{Z}_{N}$, where $\mathbb{Z}_{N}=\{0,1, \ldots, N-1\}$, we define $\mathbf{I}_{T}$ to be the vector $\left(x_{0}, x_{1}, \ldots, x_{N-1}\right)^{T}$ with $x_{i}=1$ if $i \in T$ and $x_{i}=0$ otherwise. For any nonnegative $N \times N$ row stochastic matrix $B$, i.e., the sum for each row is one, we define a map $F_{B}$ such that

$$
F_{B}(T)=\left\{j \in \mathbb{Z}_{N}:\left(B \mathbf{I}_{T}\right)_{j}=1\right\} .
$$

Let $A_{0}$ and $A_{1}$ be $N \times N$ matrices deduced by the mask $a$ :

$$
A_{0}=\left[a_{2 j-i}\right]_{0 \leq i, j \leq N-1} \text { and } A_{1}=\left[a_{2 j-i+1}\right]_{0 \leq i, j \leq N-1} .
$$

Clearly, $A_{0}$ and $A_{1}$ are row stochastic matrices if $a(1)=2$ and $a(-1)=0$. We should write simply $F_{0}=F_{A_{0}}$ and $F_{1}=F_{A_{1}}$. For convenience we should also use the following standard notation for algebraic sums of sets: let $T$ be a set of integers; then for any integers $\alpha$ and $\beta$ the set $\alpha T+\beta$ is given by

$$
\alpha T+\beta:=\{\alpha x+\beta: x \in T\} .
$$

We have (see [15])

Lemma 2.1. Let $B$ be a $N \times N$ nonnegative row stochastic matrix. Then $F_{B}\left(T_{1}\right)$ and $F_{B}\left(T_{2}\right)$ are disjoint if $T_{1}, T_{2} \subseteq \mathbb{Z}_{N}$ are. Let $C$ be an $N \times N$ nonnegative row stochastic matrix. Then $F_{B C}=F_{B} \circ F_{C}$. Furthermore, the subdivision algorithm with nonnegative mask $a$, which satisfies $a(1)=2$ and $a(-1)=0$, diverges if and only if there exist disjoint nonempty subsets $T$ and $T^{\prime}$ of $\mathbb{Z}_{N}$ and a sequence $\left(d_{1}, d_{2}, \ldots, d_{m}\right) \in\{0,1\}^{m}$ for some $m \geq 1$ such that

$$
T=F_{d_{m}} \circ \cdots \circ F_{d_{1}}(T) \text { and } T^{\prime}=F_{d_{m}} \circ \cdots \circ F_{d_{1}}\left(T^{\prime}\right) .
$$

Denote for $S(a)$ the sets $S_{0}=S(a) \cap(2 \mathbb{Z})$ and $S_{1}=S(a) \cap(2 \mathbb{Z}+1)$. The map $\Psi$ for $T \subset \mathbb{Z}$ is defined by

$$
\Psi(T)=\left\{\bigcap_{q \in S_{0}}(2 T-q)\right\} \cup\left\{\bigcap_{q \in S_{1}}(2 T-q)\right\} .
$$

The following relationship between $\Psi$ and $F_{i}$ is proved in [15]. 
Lemma 2.2. Let $a$ be a nonnegative mask satisfying $a(1)=2$ and $a(-1)=0$. Then for any $T \subset \mathbb{Z}_{N}$, we have

$$
F_{0}(T)=\Psi(T) \cap \mathbb{Z}_{N}, \quad F_{1}(T)=(\Psi(T)+1) \cap \mathbb{Z}_{N} .
$$

Furthermore, for any $\left(d_{1}, \ldots, d_{m}\right) \in\{0,1\}^{m}$ we have

$$
F_{d_{1}} \circ \cdots \circ F_{d_{m}}(T)=\left(\Psi^{m}(T)+k\right) \cap \mathbb{Z}_{N},
$$

where $k=\sum_{j=1}^{m} d_{j} 2^{j-1}$.

We may write $S(a)=\left\{0, p_{1}, \ldots, p_{k}\right\}$ with $p_{k}=N$. Generalizing Wang's lemma (see [15]), we have

Lemma 2.3. Let $T$ be a subset of $\mathbb{Z}$ and let $y \notin T$. Let $\epsilon_{i, j} \in\{0,1\}$ such that

$$
\sum_{j=1}^{k} \epsilon_{i, j} \leq 1, \quad \forall i=1,2, \ldots, m .
$$

Then it holds that

$$
2^{m} y-\sum_{j=1}^{k} \sum_{i=1}^{m} 2^{m-i} \epsilon_{i, j} p_{j} \notin \Psi^{m}(T) .
$$

Proof. For $m=1$ the assertion is clear since $2 y-x p_{l} \notin \Psi(T)$ for all $x=0,1$ and $l=1,2, \ldots, k$. On the other hand, $\epsilon_{1,1}+\cdots+\epsilon_{1, k} \leq 1$ implies either $\epsilon_{1, j}=0$ for $j=1, \ldots, k$ or else $\epsilon_{1, j}=0$ for $j \neq j^{\prime}$ and $\epsilon_{1, j^{\prime}}=1$. Thus

$$
2 y-\epsilon_{1,1} p_{1}-\epsilon_{1,2} p_{2}-\cdots-\epsilon_{1, k} p_{k} \notin \Psi(T) .
$$

The general case follows from induction on $m$.

Let $\varphi(n)$ be the Euler function of the number of elements in $\mathbb{Z}_{n}$ that are co-prime with $n$. It is known that if $\operatorname{gcd}(k, n)=1$, then

$$
k^{\varphi(n)} \equiv 1 \quad(\bmod n) .
$$

In particular, for any odd integer $n$ one has

$$
2^{\varphi(n)} \equiv 1 \quad(\bmod n) .
$$

Now we are in the position to prove the conjecture.

Proof of Conjecture. The necessity of the conditions is proved in [9]. To show the sufficiency, we denote $S(a)=\left\{0, p_{1}, \ldots, p_{k}\right\}$ with $p_{k}=N$ and $|S(a)|$ for the number of $S(a)$. The first assumption of the conjecture implies that $|S(a)| \geq 3$ and there exists an odd $p$ in $S(a)$ such that $p=p_{j_{0}}<p_{k}$ no matter if $p_{k}$ is odd or even. Hence, for $|S(a)|=3$ one has $S(a)=\{0, p, N\}$ with even $N$. Wang's result for $|S(a)|=3$ (see [15]) may be read as: the subdivision algorithm converges if $\operatorname{gcd}(p, N)=1$. We assume in the following that $|S(a)| \geq 3$. Let us observe that if the subdivision algorithms with the mask $a=\left\{a_{j}\right\}$ diverges, then by Lemma 2.1 there are a sequence $\left(d_{1}, \ldots, d_{m^{\prime}}\right)$ and disjoint nonempty sets $T, T^{\prime} \subset \mathbb{Z}_{N}$ so that

$$
F_{d_{1}} \circ \cdots \circ F_{d_{m^{\prime}}}(T)=T \text { and } F_{d_{1}} \circ \cdots \circ F_{d_{m^{\prime}}}\left(T^{\prime}\right)=T^{\prime} .
$$

It follows from Lemma 2.2 (see (2.3)) that

$$
T=\left(\Psi^{m^{\prime}}(T)+k^{\prime}\right) \cap \mathbb{Z}_{N} \quad \text { and } \quad T^{\prime}=\left(\Psi^{m^{\prime}}\left(T^{\prime}\right)+k^{\prime}\right) \cap \mathbb{Z}_{N},
$$


where $k^{\prime}=\sum_{l=1}^{m^{\prime}} d_{l} 2^{l-1}$. Write $F=F_{d_{1}} \circ \cdots \circ F_{d_{m^{\prime}}}$. Then $F^{\eta}(T)=T$ and $F^{\eta}\left(T^{\prime}\right)=$ $T^{\prime}$ for any $\eta \geq 1$. Therefore, with

$$
\begin{aligned}
k_{\eta} & =\left(\sum_{l=1}^{m^{\prime}} d_{l} 2^{l-1}\right)+\left(\sum_{l=1}^{m^{\prime}} d_{l} 2^{l-1}\right) 2^{m^{\prime}}+\cdots+\left(\sum_{l=1}^{m^{\prime}} d_{l} 2^{l-1}\right) 2^{(\eta-1) m^{\prime}} \\
& =k^{\prime} \sum_{i=0}^{\eta-1} 2^{i m^{\prime}}
\end{aligned}
$$

we get for any $\eta \geq 1$

$$
T=\left(\Psi^{m^{\prime} \eta}(T)+k_{\eta}\right) \cap \mathbb{Z}_{N} \text { and } T^{\prime}=\left(\Psi^{m^{\prime} \eta}\left(T^{\prime}\right)+k_{\eta}\right) \cap \mathbb{Z}_{N}
$$

We should choose $\eta$ in connection with $S(a)$. For our goal we define $\eta$ to be

$$
\eta=t \prod_{j=1}^{p_{k}}\{j \varphi(j)\}
$$

with some positive integer $t$, which will be given later. Denote $m=m^{\prime} \eta$. We have $2^{m} \equiv 1(\bmod x)$ for odd number $x$ from $\left\{1, \ldots, p_{k}\right\}$. Indeed, $\eta$ has a factor $x \varphi(x)$, so for some integer $n^{\prime}$ one has $\eta=n^{\prime} x \varphi(x)$. Since $x$ is odd, we obtain by Euler's formula $(2.4)$

$$
2^{m}=2^{m^{\prime} n^{\prime} x \varphi(x)} \equiv 1 \quad(\bmod x)
$$

Similarly, we have

$$
k_{\eta} \equiv 0 \quad(\bmod x) .
$$

To see this, one gets by the expression of $k_{\eta}$

$$
\begin{aligned}
k_{\eta} & =k^{\prime} \sum_{l=0}^{n^{\prime} x-1} \sum_{j=\varphi(x) l}^{\varphi(x)(l+1)-1} 2^{j m^{\prime}} \\
& =k^{\prime} \sum_{l=0}^{n^{\prime} x-1} \sum_{j=0}^{\varphi(x)-1} 2^{j m^{\prime}} 2^{\varphi(x) l m^{\prime}} \\
& \equiv k^{\prime} \sum_{l=0}^{n^{\prime} x-1} \sum_{j=0}^{\varphi(x)-1} 2^{j m^{\prime}} \quad(\bmod x) \\
& \equiv k^{\prime} n^{\prime} x \sum_{j=0}^{\varphi(x)-1} 2^{j m^{\prime}} \quad(\bmod x) \\
& \equiv 0 \quad(\bmod x),
\end{aligned}
$$

which yields (2.9). Moreover,

$$
k_{\eta}=0 \quad \Longleftrightarrow \quad d_{1}=\cdots=d_{m^{\prime}}=0
$$

and

$$
k_{\eta}=2^{m}-1 \quad \Longleftrightarrow \quad d_{1}=\cdots=d_{m^{\prime}}=1 .
$$

For all other $d_{j}$ we can thus choose $t$ large enough such that for some $0<\delta<1$

$$
p_{k}<k_{\eta}=k^{\prime} \frac{2^{m}-1}{2^{m^{\prime}}-1} \leq(1-\delta) 2^{m}
$$


and

$$
2^{m}-k_{\eta}=\frac{2^{m^{\prime} \eta}\left(2^{m^{\prime}}-1-k^{\prime}\right)+k^{\prime}}{2^{m^{\prime}}-1}>2 p_{k}
$$

Additionally, in view of (2.5) we may without loss of the generality assume that $k_{\eta}$ is even if $k_{\eta} \neq 2^{m}-1$. In the following discussion we should always denote $m$ and $k_{\eta}$ to be any nonnegative integers, which satisfy the above properties, i.e., $0 \leq k_{\eta} \leq 2^{m}$; if $k_{\eta} \neq 0,2^{m}-1$, then $k_{\eta}$ is even, $m$ and $k_{\eta}$ satisfy (2.8)-(2.11). We know that the divergence of the subdivision algorithm implies that there are two disjoint nonempty sets $T, T^{\prime} \subset \mathbb{Z}_{N}$ for (2.5). We may therefore assume $x \notin T$ and $x \in \mathbb{Z}_{N}$. So from Lemma 2.3 it holds that

$$
2^{m} x-\sum_{j=1}^{k} \sum_{i=0}^{m-1} 2^{i} \epsilon_{i, j} p_{j} \notin \Psi^{m}(T)
$$

for all $\epsilon_{i, j} \in\{0,1\}$ such that $\sum_{j=1}^{k} \epsilon_{i, j} \leq 1$. By (2.6) we obtain

$$
2^{m} x+k_{\eta}-\sum_{j=1}^{k} \sum_{i=0}^{m-1} 2^{i} \epsilon_{i, j} p_{j} \notin T
$$

for all those $\epsilon_{i, j}$. Denote $B_{0}=\{x\}$ with $x \in \mathbb{Z}_{N}$ and for $l=0,1, \ldots$,

$$
B_{l+1}=\left\{2^{m} y+k_{\eta}-\sum_{j=1}^{k} \sum_{i=0}^{m-1} 2^{i} \epsilon_{i, j} p_{j} \in \mathbb{Z}_{N} \mid y \in B_{l}, \epsilon_{i, j} \in\{0,1\}, \sum_{j=1}^{k} \epsilon_{i, j} \leq 1\right\} .
$$

Thus, (2.12) tells us that $x \notin T$ implies $B_{l} \cap T=\emptyset$ for all $l \geq 0$. In the next section we will prove the following

Lemma 2.4. Let the mask $a=\left\{a_{j}\right\}$ satisfy the conditions of the conjecture and $S(a)=\left\{0, p_{1}, \ldots, p_{k}\right\}$. Then there is an $0 \leq x<p_{k}$ such that with $B_{0}=\{x\}$ one has

$$
\bigcup_{l \geq 0} B_{l}=\mathbb{Z}_{p_{k}}
$$

This lemma, together with the above consideration, implies that the set $T$ or $T^{\prime}$ must be empty. This contradiction proves the conjecture.

The proof of Lemma 2.4 is much more involved and, in fact, is the kernel of this paper. We prove this lemma in the next section.

\section{Proof of Lemma 2.4}

The notation such as $B_{j}, k_{\eta}$ and $m$ have the same mean and properties as in Sections 1 and 2. Let us begin with the observation of the first condition of the conjecture. We need the following

Definition. A set of integers $I=\left\{0, q_{1}, \ldots, q_{k}\right\}$ with $0<q_{j}<q_{j+1}$ has property $\mathrm{P}$ if either $q_{k}$ is even and $I$ contains at least one odd integer or else $I$ contains at least two odd and two even integers (including 0 ).

Obviously, if $a=\left\{a_{j}\right\}$ satisfies the first condition of the conjecture, the set $S(a)$ has property $\mathrm{P}$. Conversely, if a set $\left\{0, q_{1}, \ldots, q_{k}\right\}$ has property $\mathrm{P}$, then one can construct a mask $a=\left\{a_{j}\right\}$ satisfying the first condition of the conjecture and 
$S(a)=\left\{0, q_{1}, \ldots, q_{k}\right\}$. In fact, in 9 ] this conjecture is formulated by using property $\mathrm{P}$. We may therefore reformulate Lemma 2.4 as

Lemma 2.4'. If $S(a)=\left\{0, p_{1}, \ldots, p_{k}\right\}$ has property $P$ and $\operatorname{gcd}\left(p_{1}, \ldots, p_{k}\right)=1$, then there is an $x \in \mathbb{Z}_{p_{k}}$ such that with $B_{0}=\{x\}$

$$
\bigcup_{l \geq 0} B_{l}=\mathbb{Z}_{p_{k}}
$$

To prove Lemma 2.4 or Lemma $2.4^{\prime}$, we remember that $\left\{\epsilon_{i, j}\right\} \in\{0,1\}$ for $i=$ $0,1, \ldots, m-1, \quad j=1, \ldots, k$ and

$$
\sum_{j=1}^{k} \epsilon_{i, j} \leq 1, \quad i=0,1, \ldots, m-1 .
$$

Let $\delta_{i, j}=\epsilon_{i, j}$ for $i=0,1, \ldots, m-1, j=1, \ldots, k-1$ and

$$
\delta_{i, k}=1-\sum_{j=1}^{k} \epsilon_{i, j} .
$$

Hence, $\left\{\delta_{i, j}\right\}$ satisfies our conditions. We obtain

$$
-\left\{2^{m}\left(p_{k}-1-x\right)+\left(2^{m}-1-k_{\eta}\right)-\sum_{j=1}^{k-1} \sum_{i=0}^{m-1} \epsilon_{i, j} 2^{i}\left(p_{k}-p_{j}\right)-\sum_{i=0}^{m-1} \epsilon_{i, k} 2^{i} p_{k}\right\} .
$$

We notice that $2^{m}-1-k_{\eta}$ is between 0 and $2^{m}-1$. Formulas (2.8) and (2.9) imply that (2.9) also holds for $k_{\eta}^{\prime}=2^{m}-1-k_{\eta}$ instead of $k_{\eta}$. Let $\hat{B}_{j}=p_{k}-1-B_{j}$. Then $\hat{B}_{j}$ is defined by $\left\{0, p_{k}-p_{k-1}, \ldots, p_{k}-p_{1}, p_{k}\right\}$ and $k_{\eta}^{\prime}$. Since $\operatorname{gcd}\left(p_{1}, \ldots, p_{k}\right)=$ $\operatorname{gcd}\left(p_{k}-p_{k-1}, \ldots, p_{k}-p_{1}, p_{k}\right)$, the symmetric relation (3.2) tells us that

$$
v \in \bigcup_{l \geq 0} B_{l} \Longleftrightarrow p_{k}-1-v \in \bigcup_{l \geq 0} \hat{B}_{l}
$$

Moreover, if $\left\{0, p_{1}, \ldots, p_{k}\right\}$ has property $\mathrm{P}$, then $\left\{0, p_{k}-p_{k-1}, \ldots, p_{k}-p_{1}, p_{k}\right\}$ does also. Thus, it is enough to show Lemma $2.4^{\prime}$ either for $\left\{0, p_{1}, \ldots, p_{k}\right\}$ or for $\left\{0, p_{k}-p_{k-1}, \ldots, p_{k}-p_{1}, p_{k}\right\}$. For simplicity we define

$$
\mathcal{B}(x)=\bigcup_{l \geq 0} B_{l}
$$

with $B_{0}=\{x\} \subset \mathbb{Z}_{p_{k}}$.

The following assertions will be used frequently and are based on some special choices of $\left\{\epsilon_{i, j}\right\}$. We formulate them as four lemmas.

Lemma 3.1. Let $y \in \mathcal{B}(x)$ and $0 \leq y \leq p_{l_{1}}$ for some odd $p_{l_{1}} \in S(a)$. If $0<$ $\left(2^{m}-1\right) y+k_{\eta} \leq\left(2^{m}-1\right) p_{l_{1}}$, then $y+j p_{l_{1}} \in \mathcal{B}(x)$ for $j$ such that $y+j p_{l_{1}} \in \mathbb{Z}_{p_{k}}$.

Proof. We choose $\epsilon_{i, j}=0$ for $j \neq l_{1}$. By the definition of $\epsilon_{i, j}$ the number $l=$ $\sum_{i=0}^{m-1} \epsilon_{i, l_{1}} 2^{i}$ can be any number between 0 and $2^{m}-1$. On the other hand, in view 
of $(2.8)$ and $(2.9)$ we have $\left(2^{m}-1\right) y+k_{\eta}=\alpha p_{l_{1}}$. Hence,

$$
y+\left(2^{m}-1\right) y+k_{\eta}-\sum_{i=0}^{m-1} \epsilon_{i, l_{1}} p_{l_{1}} 2^{i}=y+(\alpha-l) p_{l_{1}} .
$$

The choice of $\eta$ (see (2.7)) implies $k_{\eta}>p_{k}$ and $2^{m}-k_{\eta}>2 p_{k}$ if $k_{\eta} \neq 0$ and $2^{m}-1$, while for $k_{\eta}=0$, the inequality $\left(2^{m}-1\right) y>0$ yields $y \geq 1$. Moreover, if $k_{\eta}=2^{m}-1$, then the condition implies $y<p_{l_{1}}$. Hence $\alpha-l$ can be any number between $-p_{k}$ and $p_{k}$. In particular, for $l$ such that $y+(\alpha-l) p_{l_{1}} \in \mathbb{Z}_{p_{k}}$ we have $y+(\alpha-l) p_{l_{1}} \in \mathcal{B}(x)$.

Lemma 3.2. Let $y \in \mathcal{B}(x)$ and $p_{l_{1}} \in S(a)$ be even. Assume $p_{l_{1}} / 2 \leq y$ and $\left(2^{m}-1\right)\left(y-p_{l_{1}} / 2\right)+k_{\eta} \leq\left(2^{m-1}-1\right) p_{l_{2}}$ for some odd $p_{l_{2}} \in S(a)$. Then $y-p_{l_{1}} / 2 \in$ $\mathcal{B}(x)$.

Proof. We choose $\epsilon_{m-1, l_{1}}=1$ and $\epsilon_{i, l_{1}}=0$ if $i \neq m-1$. For other $j \neq l_{2}$ let $\epsilon_{i, j}=0$. To meet the condition on $\epsilon_{i, j}$, we have to choose $\epsilon_{m-1, l_{2}}=0$. Now $\sum_{i=0}^{m-2} \epsilon_{i, l_{2}} 2^{i}$ can be any number between 0 and $2^{m-1}-1$. From (2.8), (2.9) and our restriction on $y$ we conclude $\left(2^{m}-1\right)\left(y-p_{l_{1}} / 2\right)+k_{\eta}=\alpha p_{l_{2}}$ for some $0 \leq \alpha \leq 2^{m-1}-1$. Hence, we can choose $\epsilon_{i, l_{2}}$ for $i=0,1, \ldots, m-2$ to obtain $\sum_{i=0}^{m-2} \epsilon_{i, l_{2}} 2^{i}=\alpha$. With this choice of $\epsilon_{i, j}$ we get

$$
2^{m} y+k_{\eta}-\sum_{j=1}^{k} \sum_{i=0}^{m-1} \epsilon_{i, j} p_{j} 2^{i}=y-p_{l_{1}} / 2 .
$$

Thus, $y-p_{l_{1}} / 2 \in \mathcal{B}(x)$.

Lemma 3.3. Let $y \in \mathcal{B}(x)$. If there is some odd $p_{l_{1}} \in S(a)$ such that $\left(2^{m}-1\right) y+$ $k_{\eta}<\left(2^{m}-1\right) p_{l_{1}}$, then for any $p_{j^{\prime}} \in S(a)$ one has

(i) $y+(2 l+1) p_{l_{1}}-p_{j^{\prime}} \in \mathcal{B}(x)$ whenever $y+(2 l+1) p_{l_{1}}-p_{j^{\prime}} \in \mathbb{Z}_{p_{k}}$ and $\left(2^{m}-1\right) y+k_{\eta}$ is odd;

(ii) $y+2 l p_{l_{1}}-p_{j^{\prime}} \in \mathcal{B}(x)$ whenever $y+2 l p_{l_{1}}-p_{j^{\prime}} \in \mathbb{Z}_{p_{k}}$ and $\left(2^{m}-1\right) y+k_{\eta}$ is even.

Proof. Clearly $\left(2^{m}-1\right) y+k_{\eta}=\alpha p_{l_{1}}$ for some $0 \leq \alpha<2^{m}-1$. If $\alpha$ is odd, we choose $\epsilon_{i, l_{1}}$ such that $\alpha-\sum_{i=0}^{m-1} \epsilon_{i, l_{1}} 2^{i}=2 l+1$. Obviously, $\epsilon_{0, l_{1}}$ must be zero. Hence, we can choose $\epsilon_{0, j^{\prime}}=1$ or 0 to obtain

$$
2^{m} y+k_{\eta}-\sum_{j=1}^{k} \sum_{i=0}^{m-1} \epsilon_{i, j} p_{j} 2^{i}=y+(2 l+1) p_{l_{1}}-\epsilon_{0, j^{\prime}} p_{j^{\prime}} .
$$

Since $y \in \mathcal{B}(x)$, the set $\mathcal{B}(x)$ contains $y+(2 l+1) p_{l_{1}}-\epsilon_{0, j^{\prime}} p_{j}$ whenever $y+(2 l+$ 1) $p_{l_{1}}-\epsilon_{0, j^{\prime}} p_{j^{\prime}} \in \mathbb{Z}_{p_{k}}$. Similarly, we have the second assertion.

Lemma 3.4. Let $y \in \mathcal{B}(x)$ and $p_{l_{2}}-p_{l_{1}}>0$ be odd for some $p_{l_{1}}, p_{l_{2}} \in S(a)$. Assume $0<\left(2^{m}-1\right)\left(y-p_{l_{1}}\right)+k_{\eta}<\left(2^{m}-1\right)\left(p_{l_{2}}-p_{l_{1}}\right)$. Then, $y+j\left(p_{l_{2}}-p_{l_{1}}\right) \in \mathcal{B}(x)$ for $j \in \mathbb{Z}$ such that $y+j\left(p_{l_{2}}-p_{l_{1}}\right) \in \mathbb{Z}_{p_{k}}$.

Proof. Let $\epsilon_{i, l_{1}}=1-\epsilon_{i, l_{2}}$ and $\epsilon_{i, j}=0$ for $j \neq l_{1}, l_{2}$. We obtain

$$
\begin{aligned}
2^{m} y+k_{\eta}-\sum_{j=1}^{k} \sum_{i=0}^{m-1} \epsilon_{i, j} p_{j} 2^{i} & =y+\left(2^{m}-1\right)\left(y-p_{l_{1}}\right)+k_{\eta}-\sum_{i=0}^{m-1} \epsilon_{i, l_{2}}\left(p_{l_{2}}-p_{l_{1}}\right) 2^{i} \\
& =y+\left(2^{m}-1\right)\left(y-p_{l_{1}}\right)+k_{\eta}-l\left(p_{l_{2}}-p_{l_{1}}\right),
\end{aligned}
$$


where $l$ can be any integer between 0 and $2^{m}-1$. On the other hand, our condition, (2.8) and (2.9) imply

$$
p_{k} \leq\left(2^{m}-1\right)\left(y-p_{l_{1}}\right)+k_{\eta}=\alpha\left(p_{l_{2}}-p_{l_{1}}\right)<\left(2^{m}-1\right)\left(p_{l_{2}}-p_{l_{1}}\right) .
$$

Hence, for any nonnegative integer $j$ such that $y+j\left(p_{l_{2}}-p_{l_{1}}\right) \in \mathbb{Z}_{p_{k}}$ we can choose $l \in\left[0,2^{m}-1\right]$ so that $\alpha-l=j$, i.e., $y+j\left(p_{l_{2}}-p_{l_{1}}\right) \in \mathcal{B}(x)$. On the other hand, the condition implies that $y+1 \leq p_{l_{2}}$ if $k_{\eta} \neq 2^{m}-1$ and $y+2 \leq p_{l_{2}}$ if $k_{\eta}=2^{m}-1$. Thus, for any negative integer $j$ such that $y+j\left(p_{l_{2}}-p_{l_{1}}\right) \in \mathbb{Z}_{p_{k}}$ we can choose $l \in\left[0,2^{m}-1\right]$ so that $\alpha-l=j$, i.e., $y+j\left(p_{l_{2}}-p_{l_{1}}\right) \in \mathcal{B}(x)$.

We are now ready to verify Lemma $2.4^{\prime}$. We prove this lemma using induction on $|S(a)|$. The assertion (3.1) for $|S(a)|=3$ is essentially given by Wang in [15] (see the proof of Theorem 1.1 in [15]).

Proof of Lemma $2.4^{\prime}$. The idea behind the proof is that first we prove the assertion for a small $p$ instead of $p_{k}$, i.e.,

$$
\{0, \ldots, p-1\} \subseteq \mathcal{B}(x) .
$$

If $p$ is odd and belongs to $S(a)$, we can then extend this to all of $\left\{0,1, \ldots, p_{k}-1\right\}$ by using Lemma 3.1. In order to obtain the result for all of $\{0,1, \ldots, p-1\}$, we choose carefully an $x$ in this set and apply Lemmas 3.1-3.4 to get another number, which is greater than $p$. Using Lemma 3.2 or Lemma 3.4, we may reduce the number so that the new number is again contained in $\{0, \ldots, p-1\}$ but is different from $x$. Recursively, we obtain several numbers. One of our tasks is to show that those numbers are all different. A careful choice of $x$ is necessary. Otherwise the following calculation leads to $\mathcal{B}(x)=\{0\}$. In fact, if $k_{\eta}=0$, then for $x=0$ and $B_{0}=\{0\}$ one gets $B_{l}=\{0\}$ for all $l=1,2, \ldots$ Thus, in our choice of $x$ it may be understood that we avoid zero if $k_{\eta}=0$ or $p_{k}-1$ if $k_{\eta}=2^{m}-1$ during the calculation of new numbers. In other words, we should choose such an $x$ that zero or $p_{k}-1$ occurs at the last step of our calculation.

Next let us observe the set $S(a)$ with $|S(a)| \geq 5$. It is easy to check that for some nonzero $p \in S(a)$ the set $S(a) \backslash\{p\}$ also has property P. However, for sets with $|S(a)|=4$ we may not have this $p$. The only set that does not have this $p$ is $\left\{0, p_{1}, p_{2}, p_{3}\right\}$ with even $p_{1}$ and odd $p_{2}, p_{3}$. We already know that $|S(a)| \geq 3$. Since the assertion for $|S(a)|=3$ is essentially given in [15], we should omit the proof for this case. Our approach, therefore, has two steps; namely, the proof for the special case of $|S(a)|=4$ and for $|S(a)| \geq 4$. Let us begin with the special case.

Step 1. $S(a)=\left\{0, p_{1}, p_{2}, p_{3}\right\}$ with even $p_{1}$ and odd $p_{2}, p_{3}$. We divide the proof into three cases according to the different position of $p_{1}, p_{2}$ and $p_{3}$.

Case 1. Let us prove (3.1) for $2 p_{2}<p_{3}$. Assume $k_{\eta} \neq 2^{m}-1$. Denote $\operatorname{gcd}\left(p_{1}, p_{2}\right)=d$ and $p=p_{1} / 2$. The $0 \leq x<p$ is given as follows:

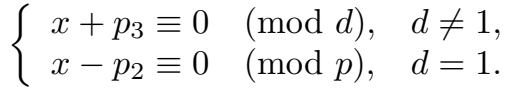

With this $x$ we have

$$
\{x+d v: 0 \leq x+d v \leq p\} \subseteq \mathcal{B}(x) .
$$

To see this, we apply Lemma 3.1 to get $x+p_{2} \in \mathcal{B}(x)$. Using Lemma 3.2 for $p_{l_{1}}=p_{1}$ and $p_{l_{2}}=p_{3}$, we conclude $x+p_{2}-p \in \mathcal{B}(x)$. Repeatedly we get $x+p_{2}-\alpha_{1} p \in$ $\mathcal{B}(x)$ whenever $0 \leq x+p_{2}-\alpha_{1} p<p$. We notice that $x+p_{2}-\alpha_{1} p$ satisfies the above restriction. Thus, recursively, i.e., replacing $x$ by $x+p_{2}-\alpha_{1} p$, we conclude 
$x+s p_{2}-\alpha_{s} p \in \mathcal{B}(x)$ and $0 \leq x+s p_{2}-\alpha_{s} p<p$ for $s=0,1, \ldots, p / d-1$, where $\alpha_{0}=0$. We note also that the choice of $x$ ensures $x+s p_{2}-\alpha_{s} p \neq 0$ at least for $s=0,1, \ldots, p / d-2$, while for $s=p / d-1$ one has

$$
\begin{cases}x+s p_{2}-\alpha_{s} p=0, & \text { if } d=1 \\ x+s p_{2}-\alpha_{s} p \neq 0, & \text { if } d \neq 1 .\end{cases}
$$

Moreover, it follows from $\operatorname{gcd}\left(p_{2}, p\right)=d$ that $x+s p_{2}-\alpha_{s} p \neq x+s^{\prime} p_{2}-\alpha_{s^{\prime}} p$ if $0 \leq s<s^{\prime} \leq p / d-1$. Therefore,

$$
\begin{aligned}
\left\{x+s p_{2}-\alpha_{s} p: s=0,1, \ldots, p / d-1\right\} & =\{x+d v: 0 \leq x+d v<p\} \\
& \subseteq \mathcal{B}(x) .
\end{aligned}
$$

Examining the way we calculate the number $x+s p_{2}-\alpha_{s} p$ for $s=p-1$ and $d=1$, we obtain additionally $p \in \mathcal{B}(x)$, proving (3.3).

Having (3.3), we will replace $p$ in $(3.3)$ by $p_{2}$. We use the same procedure as above. Let $x+d v$ be any nonzero element from $\{x+d v: 0 \leq x+d v \leq p\}$. Lemma 3.1 leads to $x+d v+p_{2} \in \mathcal{B}(x)$ while Lemma 3.2 implies $x+d v+p_{2}-l p \in \mathcal{B}(x)$ for $l \geq 0$ such that $x+d v+p_{2}-l p \in \mathbb{Z}_{p_{3}}$. Clearly, there are $l^{\prime}$ and $\nu$ such that $p_{2} / d=l^{\prime} p / d+\nu$ or $p_{2}=l^{\prime} p+\nu d$. Hence, for $p<x+d v^{\prime} \leq p_{2}$ we get $x+d v^{\prime}=x+d\left(v^{\prime}-\nu\right)+p_{2}-l^{\prime} p$. Now, if $0<x+d\left(v^{\prime}-\nu\right) \leq p$, then $x+d v^{\prime} \in \mathcal{B}(x)$. If $x+d\left(v^{\prime}-\nu\right) \leq 0$, there exists $l^{\prime \prime}$ such that $0<x+d\left(v^{\prime}-\nu\right)+l^{\prime \prime} p \leq p$. Hence, $x+d v^{\prime}=x+d\left(v^{\prime}-\nu+l^{\prime \prime} p / d\right)+p_{2}-\left(l^{\prime}+l^{\prime \prime}\right) p$. We still have $x+d v^{\prime} \in \mathcal{B}(x)$. Finally, if $p<x+d\left(v^{\prime}-\nu\right)$, one has $l^{\prime \prime \prime}$ such that $0<x+d\left(v^{\prime}-\nu\right)-l^{\prime \prime \prime} p \leq p$. Thus, $x+d v^{\prime}=x+d\left(v^{\prime}-\nu-l^{\prime \prime \prime} p / d\right)+p_{2}-\left(l^{\prime}-l^{\prime \prime \prime}\right) p$. We conclude in particular that

$$
\left\{x+d v: 0 \leq x+d v \leq p_{2}\right\} \subseteq \mathcal{B}(x) .
$$

In order to replace $p_{2}$ in (3.4) by $p_{3}-1$, we again use Lemma 3.1. Examining the conditions of Lemma 3.1, we know that if $d \neq 1$, then for each $0 \leq x+d v \leq p_{2}$ one gets $x+d v+l p_{2} \in \mathcal{B}(x)$ whenever $x+d v+l p_{2} \in \mathbb{Z}_{p_{3}}$. Hence,

$$
\left\{x+d v: 0 \leq x+d v<p_{3}\right\} \subseteq \mathcal{B}(x) .
$$

However, if $d=1$, the above calculation may not be true. We should modify this as follows: for $k_{\eta} \neq 0$ let $0 \leq x+v<p_{2}$. Again we have (3.5). If $k_{\eta}=0$, let $0<x+v \leq p_{2}$. We obtain $x+v+l p_{2} \in \mathcal{B}(x)$. Hence, (3.5) is still valid. Clearly, (3.5) implies (3.1) if $d=1$.

To show (3.1) for $d \neq 1$, we observe elements from (3.5) satisfying $p_{3}-p_{1} \leq x+$ $d v<p_{3}-p$. Obviously we can apply Lemma 3.4 for $y=x+d v, p_{l_{1}}=p_{1}$ and $p_{l_{2}}=p_{3}$ to obtain $x+d v-\left(p_{3}-p_{1}\right) \in \mathcal{B}(x)$. Noticing $2 p_{2}<p_{3}$ and $0 \leq x+d v-\left(p_{3}-p_{1}\right)<p$, we get from Lemma 3.1 that $x+d v-\left(p_{3}-p_{1}\right)+p_{2} \in \mathcal{B}(x)$ if $k_{\eta} \neq 0$ or $x-p_{3} \not \equiv 0$ $(\bmod d)$. Under this restriction, Lemma 3.2 tells us that $x+d v-\left(p_{3}-p_{1}\right)+p_{2}-p l \in$ $\mathcal{B}(x)$ whenever $x+d v-\left(p_{3}-p_{1}\right)+p_{2}-p l \in \mathbb{Z}_{p_{3}}$. Recursively, we conclude that if $k_{\eta} \neq 0$ or $x-p_{3} \neq \equiv 0(\bmod d)$, then $x+d v-\left(p_{3}-p_{1}\right)+j p_{2}-l p \in \mathcal{B}(x)$ whenever $x+d v-\left(p_{3}-p_{1}\right)+j p_{2}-l p \in \mathbb{Z}_{p_{3}}$. Let $0 \leq x+d \nu-\left(p_{3}-p_{1}\right)<p_{3}$. Then due to $\operatorname{gcd}\left(p_{2}, p\right)=d$, there are $j \geq 0, l \geq 0$ and $0 \leq x+d v-\left(p_{3}-p_{1}\right)<p$ such that

$$
x+d \nu-\left(p_{3}-p_{1}\right)=x+d v-\left(p_{3}-p_{1}\right)+j p_{2}-l p .
$$

Therefore, if $k_{\eta} \neq 0$ or $x-p_{3} \neq \equiv 0(\bmod d)$,

$$
\left\{x+d v-\left(p_{3}-p_{1}\right): 0 \leq x+d v-\left(p_{3}-p_{1}\right)<p_{3}\right\} \subseteq \mathcal{B}(x) .
$$


This observation leads to the following assertion: Let $A_{j}=\left\{x+d v-j\left(p_{3}-p_{1}\right)\right.$ : $\left.0 \leq x+d v-j\left(p_{3}-p_{1}\right)<p_{3}\right\}$. Then under the assumption $k_{\eta} \neq 0$ or $x-(i+1) p_{3} \neq \equiv 0$ $(\bmod d)$ we have that $A_{i} \subseteq \mathcal{B}(x)$ implies $A_{i+1} \subseteq \mathcal{B}(x)$. We already know $A_{0} \cup A_{1} \subseteq$ $\mathcal{B}(x)$. Thus, we use the above procedure with $p_{3}-p_{1} \leq x+d v-i\left(p_{3}-p_{1}\right)<p_{3}-p$ instead of $p_{3}-p_{1} \leq x+d v-(i-1)\left(p_{3}-p_{1}\right)<p_{3}-p$ to obtain $A_{i+1} \subset \mathcal{B}(x)$. We remember $x+p_{3} \equiv 0(\bmod d)$. Hence, $x-j p_{3} \not \equiv 0(\bmod d)$ for $j=0,1, \ldots, d-2$. We conclude

$$
\bigcup_{j=0}^{d-2} A_{j} \subseteq \mathcal{B}(x)
$$

Moreover, in the case of $k_{\eta} \neq 0$

$$
\bigcup_{j=0}^{d-1} A_{j} \subseteq \mathcal{B}(x) .
$$

To have (3.1) for $k_{\eta} \neq 0$, we notice $\operatorname{gcd}\left(d, p_{3}\right)=1$. Hence, for any $v^{\prime}$ such that $0 \leq x+v^{\prime}<p_{3}$ there is $0 \leq j \leq d-1$ with $v^{\prime}+j\left(p_{3}-p_{1}\right) \equiv 0(\bmod d)$, which gives $v^{\prime}=d v-j\left(p_{3}-p_{1}\right)$ for some $v$. We conclude that $x+v^{\prime}=x+d v-j\left(p_{3}-p_{1}\right) \in A_{j}$ or

$$
\mathbb{Z}_{p_{3}}=\left\{v: 0 \leq v<p_{3}\right\} \subseteq \mathcal{B}(x) .
$$

It remains to show (3.1) for $k_{\eta}=0$. We already have (3.6). But what is the corresponding $A_{d-1}$ for this case? We know that the first step in our procedure is to find an element in $A_{d-2}$ which satisfies

$$
p_{3}-p_{1} \leq x+d v-(d-2)\left(p_{3}-p_{1}\right)<p_{3}-p .
$$

If $x+d v-(d-2)\left(p_{3}-p_{1}\right) \neq p_{3}-p_{1}$, we can use our procedure to obtain $x+d v-$ $(d-1)\left(p_{3}-p_{1}\right)+j p_{2}-l p \in \mathcal{B}(x)$. If however $x+d v-(d-2)\left(p_{3}-p_{1}\right)=p_{3}-p_{1}$, we get formally from the above procedure a set

$$
A^{\prime}=\left\{j p_{2}-l p: 0 \leq j p_{2}-l p<p_{3}\right\} .
$$

But, because $k_{\eta}=0$, we cannot use this approach for $x+d v-(d-1)\left(p_{3}-p_{1}\right)(=0)$. Thus, instead of $A_{d-1}$ we have only $A_{d-1} \backslash A^{\prime} \subset \mathcal{B}(x)$. Hence, combining this with (3.6), we obtain $\left(\bigcup_{i=0}^{d-1} A_{i}\right) \backslash A^{\prime} \subseteq \mathcal{B}(x)$, which implies

$$
\left\{v: 0 \leq v<p_{3}\right\} \backslash A^{\prime} \subseteq \mathcal{B}(x) .
$$

To show $A^{\prime} \subset \mathcal{B}(x)$, we have to modify (3.7). We remember that $\operatorname{gcd}(d, p)=d$ and $x-(d-1) p_{3} \equiv 0(\bmod d)$. Hence, there is a $v^{\prime}$ such that

$$
p_{3}-p_{1}<x+d v^{\prime}-(d-2)\left(p_{3}-p_{1}\right)=p_{3}-p,
$$

which implies that $x+d v^{\prime}-(d-1)\left(p_{3}-p_{1}\right)(=p)$ is contained in $\mathcal{B}(x)$ due to Lemma 3.4. Now we can apply our procedure for $p$ to obtain $j p_{2}-l p \in \mathcal{B}(x)$ or $A^{\prime} \subset \mathcal{B}(x)$. Consequently,

$$
\mathbb{Z}_{p_{3}}=\left\{v: 0 \leq v<p_{3}\right\} \subseteq \mathcal{B}(x) .
$$

For $k_{\eta}=2^{m}-1$ we replace $x+p_{3} \equiv 0(\bmod d)$ by $x+1+p_{3} \equiv 0(\bmod d)$ and $p \mid\left(x-p_{2}\right)$ by $p \mid\left(x+1-p_{2}\right)$, respectively.

Case 2. Now if $p_{3}<2 p_{1}$, we use the symmetric relation to obtain (3.1), since $\left(0, p_{3}-p_{2}, p_{3}-p_{1}, p_{3}\right)$ has property $\mathrm{P}$ and $2\left(p_{3}-p_{1}\right)<p_{3}$. 
Case 3. Since $p_{3}$ is odd, it remains to show (3.1) for $2 p_{1}<p_{3}<2 p_{2}$. Without loss of generality we may suppose $p_{3} \geq p_{2}+p_{1}$. Now, $p_{1}$ is even, so $p_{1}=2 p$ for some $p$.

Let $p$ be odd. Denote $\operatorname{gcd}\left(p_{1}, p_{3}\right)=d$. We have $\operatorname{gcd}\left(p, p_{3}\right)=d$. We verify for some $0 \leq x \leq p$ that

$$
\left\{v: 0 \leq v<p_{1}\right\} \backslash\{p\} \subseteq \mathcal{B}(x) .
$$

To this end we choose $0 \leq x \leq p$ in the following way: if $0 \leq k_{\eta}<2^{m}-1$, then $x \neq 0$ and

$$
\left\{\begin{array}{lll}
x-p_{2} \equiv 0 & (\bmod d), & \text { if } d \neq 1, \\
x+p_{3} \equiv 0 & (\bmod p), & \text { if } d=1 ;
\end{array}\right.
$$

if $k_{\eta}=2^{m}-1$, then $x \neq p$ and

$$
\begin{cases}x+1-p_{2} \equiv 0 \quad(\bmod d), & \text { if } d \neq 1 \\ x+1 \equiv 0(\bmod p), & \text { if } d=1 .\end{cases}
$$

In the following we deal only with the case $0 \leq k_{\eta}<2^{m}-1$. The corresponding assertion for $k_{\eta}=2^{m}-1$ can be treated in the same way.

To begin with we note $p \mid\left(2^{m}-1\right)$ and $p \mid k_{\eta}$ (see (2.8) and (2.9)). Hence, for some $k$ we get $2^{m} x+k_{\eta}=x+k p$. Since $k_{\eta}<(1-\delta) 2^{m}$ for some $0<\delta<1$ (see (2.10)), the number $k$ satisfies $2 p_{3}<k<(2-\delta) 2^{m}$. Write $k=2 \mu+\beta$ with $\beta \in\{0,1\}$, so $p_{3}<\mu<(1-\delta / 2) 2^{m}$. Let $\epsilon_{i, j}=0$ for $j=2,3$. We get

$$
\begin{aligned}
2^{m} x+k_{\eta}-\sum_{i=0}^{m-1} \sum_{j=1}^{3} \epsilon_{i, j} p_{j} 2^{i} & =2^{m} x+k_{\eta}-l p_{1} \\
& =x+k p-l p_{1} \\
& =x+\mu p_{1}-l p_{1}+\beta p \\
& =x+\alpha p_{1}+\beta p .
\end{aligned}
$$

We can choose $l$ satisfying $p_{3}-p_{1} \leq x+\alpha p_{1}+\beta p<p_{3}$. Thus, $x+\alpha p_{1}+\beta p \in \mathcal{B}(x)$ by the definition of $\mathcal{B}(x)$. Now the fact $p_{1}<p_{3}-p_{1}$ and Lemma 3.4 imply further

$$
0 \leq x+\alpha p_{1}+\beta p-p_{3}+p_{1}<p_{1} \text { and } x+\alpha p_{1}+\beta p-p_{3}+p_{1} \in \mathcal{B}(x) \text {. }
$$

Lemma 3.2 tells us that we can substitute $p$ if the above number is not less than $p$. We obtain in this way $x-p_{3}+l_{1} p=x+\alpha p_{1}+\beta p-p_{3}+p_{1}-p \in \mathcal{B}(x)$ and $0 \leq x-p_{3}+l_{1} p<p$. As in Case 1 we conclude recursively that $0 \leq x-s p_{3}+l_{s} p<p$ for $s=0, \ldots, p / d-1$. Clearly, by this construction $x-s p_{3}+l_{s} p \in \mathcal{B}(x)$. Moreover, since $\operatorname{gcd}\left(p, p_{3}\right)=d$, all these numbers are different for $0 \leq s \leq p / d-1$. Hence,

$$
\begin{aligned}
\left\{x-s p_{3}+l_{s} p: x-s p_{3}+l_{s} p \in[0, p)\right\} & =\{x+d v: 0 \leq x+d v<p\} \\
& \subseteq \mathcal{B}(x) .
\end{aligned}
$$

We notice also that the set on the left-hand side contains the zero if and only if $d=1$. For $d=1$ we conclude from the last relation that

$$
\{0,1, \ldots, p-1\} \subseteq \mathcal{B}(x) .
$$

We claim that (3.11) is still valid for $d \neq 1$. To see this, let $0<x+d v<p$. The above consideration tells us that we have $\alpha$ and $\beta$, where $\beta \in\{0,1\}$ depends on $x+d v$, such that $x+d v+\alpha p_{1}+\beta p \in \mathcal{B}(x)$ and $p_{3}-p_{1}-p_{2} \leq x+d v+$ $\alpha p_{1}+\beta p<p_{3}-p_{2}$. Now, since $d \neq 1$, we deduce $p_{3}-p_{1}-p_{2} \neq 0$. Hence, $0<x+d v+\alpha p_{1}+\beta p<p_{3}-p_{2}<p_{2}$. We can therefore apply Lemma 3.1 for 
$x+d v+\alpha p_{1}+\beta p$ to obtain $x+d v+\alpha p_{1}+\beta p+p_{2} \in \mathcal{B}(x)$ and by Lemma 3.4 we have $x+d v+\alpha p_{1}+\beta p+p_{2}-p_{3}+p_{1} \in \mathcal{B}(x)$. This number is nonnegative and less than $p_{1}$. So by Lemma 3.2 we can again substitute $p$, if necessary, to obtain $x+d v+\left(p_{2}-p_{3}+p_{1}\right)+l_{1} p \in \mathcal{B}(x)$ and $0 \leq x+d v+\left(p_{2}-p_{3}+p_{1}\right)+l_{1} p<p$. Recursively, $\mathcal{B}(x)$ contains

$$
x+d v+s\left(p_{2}-p_{3}+p_{1}\right)+l_{s, v} p=x+d v+s p_{2}+l_{s, v}^{\prime} d, \quad \forall s=0,1, \ldots, d-1 .
$$

Since $\operatorname{gcd}\left(p_{2}, d\right)=1$, we conclude by the second expression that they numbers are different for different $s$, while the first one shows that they are different if $v$ is different. Thus, there are $d \times p / d=p$ numbers in $\{0, \ldots, p-1\}$, which are contained in $\mathcal{B}(x)$; in other words, (3.11) also holds for $d \neq 1$.

To get (3.8) from (3.11), we observe any nonzero number $\mu$ satisfying $0<\mu<p$. We know that $\mu+\alpha p_{1}+\beta p \in \mathcal{B}(x)$ whenever $0 \leq \mu+\alpha p_{1}+\beta p<p_{3}$, where $\beta \in\{0,1\}$ relies on $\mu$. For the $\mu$ with $\beta=0$ let $\alpha=1$. So $\mu+p_{1} \in \mathcal{B}(x)$ and by Lemma 3.2, $\mu+p_{1}-p \in \mathcal{B}(x)$. For the $\mu$ with $\beta=1$ we choose $\alpha=0$. Hence, we always have $\mu+p \in \mathcal{B}(x)$, which implies (3.8).

To verify our assertion from (3.8), we remember that $0 \leq k_{\eta}<2^{m}-1$. So $k_{\eta}$ is even. If $0<k_{\eta}<2^{m}-1$, then since $0 \in \mathcal{B}(x)$ and $2^{m} 0+k_{\eta}-l p_{1}=j p_{1}$ for some $l$, we conclude $j p_{1} \in \mathcal{B}(x)$ if $0 \leq j p_{1}<p_{3}$. Lemma 3.2 yields $p \in \mathcal{B}(x)$. Combining this with (3.8), we obtain

$$
\left\{0,1, \ldots, p_{1}-1, p_{1}, 2 p_{1}, \ldots, j_{0} p_{1}\right\} \subseteq \mathcal{B}(x),
$$

where $j_{0}$ satisfies $j_{0} p_{1}<p_{3} \leq\left(j_{0}+1\right) p_{1}$. Moreover, for any $0<2 l<p_{1}$ we have $2^{m} 2 l+k_{\eta}-i p_{1}=2 l+\alpha p_{1}$ with some $i$. Hence, $\mathcal{B}(x)$ contains $2 l+\alpha p_{1}$ whenever $0<2 l+\alpha p_{1}<p_{3}$. In particular,

$$
\left\{0,1, \ldots, p_{1}\right\} \cup\left\{2 j: 0 \leq 2 j<p_{3}\right\} \subseteq \mathcal{B}(x) .
$$

Let $0<2 j<p_{2}$. Then Lemma 3.1 shows that $2 j+p_{2} \in \mathcal{B}(x)$ whenever $0<$ $2 j+p_{2}<p_{3}$. Since $p_{2}$ is odd and $2 p_{2}>p_{3}$, we obtain from (3.12) that

$$
\left\{l: p_{2}<l<p_{3}\right\} \subseteq \mathcal{B}(x) .
$$

Let $p_{1}+p_{2} \leq 2 j<p_{3}$. Lemma 3.3 tells us $2 j-p_{2} \in \mathcal{B}(x)$. It follows from (3.8) and the last two relations that

$$
\left\{l: 0 \leq l \leq p_{3}-p_{2}\right\} \subseteq \mathcal{B}(x) .
$$

Let $l$ be odd and let it satisfy $0 \leq l<p_{3}-p_{2}$. So using (i) of Lemma 3.3 several times, we obtain $l+j\left(p_{3}-p_{2}\right) \in \mathcal{B}(x)$ whenever $0 \leq l+j\left(p_{3}-p_{2}\right)<p_{3}$. We conclude from the last relation that

$$
\left\{l: 0<l<p_{3}, \quad l \text { is odd }\right\} \subseteq \mathcal{B}(x) .
$$

Assertion (3.1) follows from (3.12) and (3.13).

Next we prove (3.1) for $k_{\eta}=0$ via (3.8). The above approach tell us that we need only to have (3.12). For this goal let us observe $\mathcal{B}\left(p_{1}\right)$. By the definition of $\mathcal{B}\left(p_{1}\right)$ we certainly have $\left\{l p_{1}: 0<l \leq l^{\prime}\right\} \subseteq \mathcal{B}\left(p_{1}\right)$, where $l^{\prime}$ satisfies $l^{\prime} p_{1}<p_{3} \leq\left(l^{\prime}+1\right) p_{3}$. Lemma 3.2 implies $p \in \mathcal{B}\left(p_{1}\right)$. On the other hand, there is a choice of $\epsilon_{i, j}$ such that

$$
2^{m} p_{1}-\sum_{i=0}^{m-1} \sum_{j=1}^{3} \epsilon_{i, j} 2^{i} p_{j}=\left(2^{m}-1\right) p_{1}-\sum_{i=1}^{m-1} \epsilon_{i, 3} 2^{i} p_{3}=0 .
$$

Thus,

$$
\left\{l p_{1}: 0 \leq l \leq l^{\prime}\right\} \cup\{p\} \subseteq \mathcal{B}\left(p_{1}\right) .
$$


Let $p_{3}-p_{1} \leq l p_{1}<p_{3}$. It follows from Lemma 3.4 that $(l+1) p_{1}-p_{3} \in \mathcal{B}\left(p_{1}\right)$. Thus, by Lemma 3.2 there exists a $j$ such that $0<j p-p_{3} \leq p$ and $j p-p_{3} \in$ $\mathcal{B}\left(p_{1}\right)$. Similarly, let $p_{3}-p_{2}-p_{1}<l p_{1} \leq p_{3}-p_{2}$. We conclude for some $j$ that $j p-p_{3}+p_{2} \in \mathcal{B}\left(p_{1}\right)$ and $0<j p-p_{3}+p_{2} \leq p$. Now we can choose $x$ as follows:

$$
\begin{cases}x=j p-p_{3}+p_{2} \text { and } 0<j p-p_{3}+p_{2} \leq p, & \text { if } d \neq 1, \\ x=j p-p_{3} \text { and } 0<j p-p_{3} \leq p, & \text { if } d=1 .\end{cases}
$$

Clearly, $x$ satisfies (3.9) and $x \in \mathcal{B}\left(p_{1}\right)$. Consequently, (3.8) holds with this $x$ and $\mathcal{B}(x) \subseteq \mathcal{B}\left(p_{1}\right)$. We obtain by (3.8) the desired relation, i.e.,

$$
\left\{0,1, \ldots, p_{1}-1, p_{1}, 2 p_{1}, \ldots, l^{\prime} p_{1}\right\} \subseteq \mathcal{B}\left(p_{1}\right) .
$$

Now let $p=p_{1} / 2$ be even. Denote $\operatorname{gcd}\left(p_{3}-p_{2}, p\right)=d$. So $d$ is even. We verify for some $0 \leq x<p$ that

$$
\{x+d v: 0 \leq x+d v<p\} \subseteq \mathcal{B}(x) .
$$

To this end we choose $0 \leq x<p$ as follows:

$$
\begin{cases}x-p_{2} \equiv 0 \quad(\bmod d), & \text { if } 0 \leq k_{\eta}<2^{m}-1, \\ x+1-p_{2} \equiv 0 \quad(\bmod d), & \text { if } k_{\eta}=2^{m}-1 .\end{cases}
$$

Clearly, $x$ is not unique. But $x$ is odd if $0 \leq k_{\eta}<2^{m}-1$ and even when $k_{\eta}=2^{m}-1$. Moreover, any $0 \leq x+d v<p$ satisfies (3.15). To verify (3.14), we remember that $k_{\eta}$ is even when $0 \leq k_{\eta}<2^{m}-1$. So in any case $\left(2^{m}-1\right) x+k_{\eta}$ is odd. We also remember $p_{3} \mid\left(2^{m}-1\right)$ and $p_{3} \mid k_{\eta}$ since $p_{3}$ is odd. Now by Lemma 3.3 we obtain $x+p_{3}-p_{2} \in \mathcal{B}(x)$. On the other hand, since $x-p \leq-1$ and $p_{3}-p_{2} \leq\left(p_{3}-1\right) / 2$, we have

$$
\left(2^{m}-1\right)\left(x+p_{3}-p_{2}-p\right)+k_{\eta}<\left(2^{m-1}-1\right) p_{3} .
$$

Thus, the condition of Lemma 3.2 is satisfied. We obtain by Lemma 3.2 with $y=x+p_{3}-p_{2}, p_{l_{1}}=p_{1}$ and $p_{l_{2}}=p_{3}$ that $x+p_{3}-p_{2}-p \in \mathcal{B}(x)$. Recursively, $x+p_{3}-p_{2}-\alpha_{1} p \in \mathcal{B}(x)$ for some $\alpha_{1}$ such that $0 \leq x+p_{3}-p_{2}-\alpha_{1} p<p$. Clearly, this new number also satisfies (3.15). We can therefore apply this procedure for this new number to obtain another number that satisfies (3.15) and is contained in $\mathcal{B}(x)$. Repeatedly, we obtain for $s=0,1, \ldots, p / d-1$ that

$$
x+s\left(p_{3}-p_{2}\right)-\alpha_{s} p \in \mathcal{B}(x) \text { and } 0 \leq x+s\left(p_{3}-p_{2}\right)-\alpha_{s} p<p,
$$

where $\alpha_{0}=0$. Since $\operatorname{gcd}\left(p_{3}-p_{2}, p\right)=d$, they are all different and have the form $x+d v$. This shows (3.14).

Next we verify

$$
\{0, \ldots, p-1\} \subseteq \mathcal{B}(x) .
$$

To show (3.16), let $0 \leq x+d v<p$. Since $\left(2^{m}-1\right)(x+d v)+k_{\eta}$ is odd, it follows from (i) of Lemma 3.3 with $p_{j^{\prime}}=p_{1}$ and $p_{l_{1}}=p_{2}$ that $x+d v+p_{2}-p_{1} \in \mathcal{B}(x)$. Now $\left(2^{m}-1\right)\left(x+d v+p_{2}-p_{1}\right)+k_{\eta}$ is even. We apply (ii) of Lemma 3.3 with $p_{l_{1}}=p_{2}$ and, if necessary, Lemma 3.3 with $p_{l_{1}}=p_{1}$ to obtain $0 \leq x+d v+p_{2}-l_{1} p<p$ and

$$
x+d v+p_{2}-p_{1}-l p=x+d v+p_{2}-l_{1} p \in \mathcal{B}(x) .
$$

For this new number $\left(2^{m}-1\right)\left(x+d v+p_{2}-l_{1} p\right)+k_{\eta}$ is even. Clearly, we can again use (ii) of Lemma 3.3 to obtain $x+d v+p_{2}-l_{1} p+2 p_{2}-p_{3} \in \mathcal{B}(x)$. Write

$$
x+d v+p_{2}-l_{1} p+2 p_{2}-p_{3}=x+d v+2 p_{2}-l_{1} p-\left(p_{3}-p_{2}\right) .
$$


For this number $\left(2^{m}-1\right)\left(x+d v+2 p_{2}-l_{1} p-\left(p_{3}-p_{2}\right)\right)+k_{\eta}$ is odd. We get by (i) of Lemma 3.3 and, if necessary, Lemma 3.2 that $0 \leq x+d v+2 p_{2}-l_{2} p-l^{\prime}\left(p_{3}-p_{2}\right)<p$ and

$$
x+d v+2 p_{2}-l_{1} p-l^{\prime}\left(p_{3}-p_{2}\right)-l p=x+d v+2 p_{2}-l_{2} p-l^{\prime}\left(p_{3}-p_{2}\right) \in \mathcal{B}(x) .
$$

Repeatedly, we conclude for $s=0,1, \ldots, d-1$ that

$0 \leq x+d v+s p_{2}-l_{s} p-l_{s}^{\prime}\left(p_{3}-p_{2}\right)<p$ and $x+d v+s p_{2}-l_{s} p-l_{s}^{\prime}\left(p_{3}-p_{2}\right) \in \mathcal{B}(x)$.

By our construction it is easy to see that if $(v, s) \neq\left(v^{\prime}, s^{\prime}\right)$, then the corresponding numbers are different. There are $p / d$ different $v$ and $d$ different $s$. Therefore, $\mathcal{B}(x)$ contains $d \times p / d$ numbers in $\{0, \ldots, p-1\}$. In other words, (3.16) holds.

We have to show that $p$ in (3.16) can be replaced by $p_{3}$. We first prove this for $k_{\eta} \neq 2^{m}-1$. Let $0<l<p$ be odd. So $\left(2^{m}-1\right) l+k_{\eta}$ is odd. As before we use Lemmas 3.3 and 3.2 to obtain $l+p_{3}-p_{2}-\alpha p \in \mathcal{B}(x)$ for $\alpha \geq 0$ satisfying $0 \leq l+p_{3}-p_{2}-\alpha p<p_{3}$. Since $l+p_{3}-p_{2}-\alpha p=l^{\prime}$ is again odd, we obtain in particular that all odd numbers in $\left\{0, \ldots, p_{3}-p_{2}-1\right\}$ belong to $\mathcal{B}(x)$. Applying Lemma 3.3 for each odd number $l^{\prime}$ from the last set, we conclude $l^{\prime}+l\left(p_{3}-p_{2}\right) \in \mathcal{B}(x)$ whenever $0 \leq x+d v+l\left(p_{3}-p_{2}\right)<p_{3}$ and $l \geq 0$. Thus, $\mathcal{B}(x)$ contains all odd $0<l^{\prime}<p_{3}$, i.e.

$$
\left\{l: \quad 0 \leq l<p_{3}, l \text { is odd }\right\} \subseteq \mathcal{B}(x) .
$$

Let $l$ be odd and $0<l<p_{3}-p_{2}$. Since $p_{3}-p_{2}<p_{2}$, we have by Lemma 3.1 with $p_{2}$ that $l+p_{2} \in \mathcal{B}(x)$. Consequently,

$$
\left\{l: p_{2} \leq l<p_{3}\right\} \subseteq \mathcal{B}(x) .
$$

Furthermore, let $l$ be even and $p_{2} \leq l<p_{3}$. So $\left(2^{m}-1\right) l+k_{\eta}$ is even. We conclude from Lemma 3.3 that $\mathcal{B}(x)$ contains $l-\mu p_{1}$ whenever $\mu \geq 0$ and $0 \leq l-\mu p_{1}<p_{3}$. It follows from the fact that $p_{3} \geq p_{2}+p_{1}$ and $p_{1}$ is even that

$$
\left\{l: 0 \leq l<p_{2}, l \text { is even }\right\} \subseteq \mathcal{B}(x) .
$$

The desired assertion follows from (3.16)-(3.19).

The proof for $k_{\eta}=2^{m}-1$ is the same. We omit the details here.

Step 2. Let (3.1) be true for $|S(a)| \leq k$ with $k \geq 3$. We verify (3.1) for $|S(a)|=$ $k+1$. Write $S(a)=\left\{0, p_{1}, \ldots, p_{k}\right\}$ and $\tilde{S}(a)=\left\{0, p_{k}-p_{k-1}, \ldots, p_{k}-p_{1}, p_{k}\right\}$. Therefore, we need to prove (3.1) either for $S(a)$ or for $\tilde{S}(a)$. The special case of $|S(a)|=4$, which we dealt with in Step 1, allows us to suppose that there is $0<$ $p \in S(a)$ such that $S(a) \backslash\{p\}$ has property P. Denote $\operatorname{gcd}\left(p^{\prime}: p^{\prime} \in S(a) \backslash\{p\}\right)=d$ and

$$
S^{\prime}(a)=\{q: d q \in S(a) \backslash\{p\}\}=\left\{0, q_{1}, \ldots, q_{k-1}\right\} .
$$

$S^{\prime}(a)$ has property P. Moreover, $\operatorname{gcd}\left(q_{1}, \ldots, q_{k-1}\right)=1$ and $\left|S^{\prime}(a)\right|=k$. Let us also remark that in general $m$ and $k_{\eta}$ depend on $S(a)$. Hence, for different $S(a)$ the numbers $m$ and $k_{\eta}$ are generally different. On the other hand, it is clear that if $m$ and $k_{\eta}$ are numbers for $S(a)$, they are also suitable for $S(a) \backslash\{p\}$. Furthermore, $m$ and $k_{\eta} / d$ are numbers for $S^{\prime}(a)$. With this in mind it is now clear that the hypothesis of the induction implies for some $0 \leq x^{\prime}<q_{k-1}$ that

$$
\left\{0, \ldots, q_{k-1}-1\right\}=\mathcal{B}^{\prime}\left(x^{\prime}\right),
$$


where $\mathcal{B}^{\prime}\left(x^{\prime}\right)$ is the corresponding set $\mathcal{B}(x)$ defined by $S^{\prime}(a), m$ and $k_{\eta} / d$. By Lemma 3.1 and the definition of $\mathcal{B}\left(d x^{\prime}\right)$ we obtain

$$
\left\{l d: 0 \leq l d<p_{k}\right\} \subseteq \mathcal{B}\left(d x^{\prime}\right) .
$$

Indeed, if $d q_{k-1}=p_{k},(3.20)$ follows directly from the definition of $\mathcal{B}\left(d x^{\prime}\right)$. If however $d q_{k-1} \neq p_{k}$, then $p=p_{k}$ and $d q_{k-1}=p_{k-1}$. So

$$
\left\{l d: 0 \leq l d<p_{k-1}\right\} \subseteq \mathcal{B}\left(d x^{\prime}\right) .
$$

But there is an odd number in $S(a) \backslash\left\{p_{k}, p_{k-1}\right\}$. Using Lemma 3.1 with this number, we can still replace $p_{k-1}$ by $p_{k}$. Clearly, (3.20) is (3.1) if $d=1$. So in the following discussion we should always assume $d \neq 1$. In what follows we should use (3.20) to prove (3.1) for $S(a)$. The proof will be divided into four cases according to different $p$ and $p_{k}$.

Case 1. $p_{k}$ is even and there are at least two odd numbers in $S(a)$. Without loss of generality let two odd numbers be $p_{1}$ and $p_{2}$. Let $\operatorname{gcd}\left(p_{1}, p_{3}, \ldots, p_{k}\right)=d$ and

$$
S^{\prime}(a)=\left\{q_{i}: d q_{i} \in S(a) \backslash\left\{p_{2}\right\}\right\}=\left\{0, q_{1}, \ldots, q_{k-1}\right\} .
$$

We obtain (3.20) with $p=p_{2}$. To verify (3.1) for $S(a)$ from (3.20), let $2 p_{2} \leq p_{k}$. We choose $l^{\prime} \geq 0$ such that $p_{k}-p_{2}+l d \leq l^{\prime} d+l d<p_{k}-p_{2}+(l+1) d$ for $l=0, \ldots, p_{1} / d-1$. Clearly, $l^{\prime} d+l d \in \mathcal{B}\left(d x^{\prime}\right)$. Moreover, by Lemma 3.4 the set $\mathcal{B}\left(d x^{\prime}\right)$ contains $l^{\prime} d+l d-p_{k}+p_{2}$ and

$$
l d \leq l^{\prime} d+l d-p_{k}+p_{2}<(l+1) d \leq p_{1} .
$$

Denote $y=l^{\prime} d-p_{k}+p_{2}$. We have $y-p_{2} \equiv 0(\bmod d)$ and $y \neq 0$. Using Lemma 3.1, we conclude that for some $\alpha_{l, 1}>0$ and $l=0, \ldots, p_{1} / d-1$ the numbers $y+l d+\alpha_{l, 1} p_{1}$ are in $\mathcal{B}\left(d x^{\prime}\right)$ as well as in $\left[p_{k}-p_{2}, p_{k}-p_{2}+p_{1}\right)$. Again by Lemma 3.4 we obtain for $l=0,1, \ldots, p_{1} / d-1$ that

$$
0<y+l d+\alpha_{l, 1} p_{1}-\left(p_{k}-p_{2}\right)<p_{1} \text { and } y+l d+\alpha_{l, 1} p_{1}-\left(p_{k}-p_{2}\right) \in \mathcal{B}\left(d x^{\prime}\right) .
$$

We note that the choice of $y$ ensures $y-s\left(p_{k}-p_{2}\right) \equiv 0(\bmod d)$ if and only if $s+1 \equiv 0(\bmod d)$. Thus, write $\alpha_{l, 0}=0$. We conclude repeatedly that $\mathcal{B}\left(d x^{\prime}\right)$ contains $y+l d+\alpha_{l, s} p_{1}-s\left(p_{k}-p_{2}\right)$ and

$$
0 \leq y+l d+\alpha_{l, s} p_{1}-s\left(p_{k}-p_{2}\right)<p_{1}, \quad \forall s=0, \ldots, d-1, l=0,1, \ldots, p_{1} / d-1 .
$$

It is easy to see that all these numbers are different. Consequently, $\left\{0, \ldots, p_{1}-1\right\} \subseteq$ $\mathcal{B}\left(d x^{\prime}\right)$. Since $d \mid p_{1}$, we get by (3.20) that

$$
\left\{0, \ldots, p_{1}\right\} \cup\left\{l d: 0 \leq l d<p_{k}\right\} \subseteq \mathcal{B}\left(d x^{\prime}\right) .
$$

The desired assertion follows from this relation and Lemma 3.1, since any $p_{1}<z<$ $p_{k}$ can be written as $z=z^{\prime}+\nu p_{1}$ with $0 \leq z^{\prime}<p_{1}$.

Let $2 p_{1} \geq p_{k}$. Then $2\left(p_{k}-p_{k-2}\right) \leq p_{k}$. Hence, the above proof is valid for $\tilde{S}(a)$. We again get (3.1) by the symmetric relation (3.2).

Finally, let $2 p_{1}<p_{k}<2 p_{2}$. Equation (3.2) allows us to suppose $p_{1}+p_{2} \leq p_{k}$. The approach is the same as for the case $2 p_{2} \leq p_{k}$. First we choose $l^{\prime} \geq 0$ such that

$$
p_{k}-p_{2}-p_{1}+l d \leq l^{\prime} d+l d<p_{k}-p_{2}-p_{1}+(l+1) d, \quad \forall l=0, \ldots, p_{1} / d-1 .
$$

Hence, by Lemma 3.1 and (3.20) the number $l^{\prime} d+p_{2}$ is contained in $\mathcal{B}\left(d x^{\prime}\right)$, while Lemma 3.4 implies that $y=l^{\prime} d+p_{2}+p_{1}-p_{k}$ is in $\mathcal{B}\left(d x^{\prime}\right)$. Moreover, for the same reason

$$
y+l d \in \mathcal{B}\left(d x^{\prime}\right) \text { and } l d \leq y+l d<(l+1) d, \quad \forall l=0, \ldots, p_{1} / d-1 .
$$


Clearly, $y+s p_{2} \equiv 0(\bmod d)$ if and only if $s+1 \equiv 0(\bmod d)$. Therefore, using the same approach as for $2 p_{2} \leq p_{k}$, we conclude that $\mathcal{B}\left(d x^{\prime}\right)$ contains $y+d l+\alpha_{l, s} p_{1}-$ $s\left(p_{k}-p_{2}-p_{1}\right)$ for $s=0,1, \ldots, d-1$ and $l=0, \ldots, p_{1} / d-1$. These numbers are different and are in $\left[0, p_{1}\right)$. Consequently, $\left\{0, \ldots, p_{1}\right\} \cup\left\{l d: 0 \leq l d<p_{k}\right\} \subseteq \mathcal{B}\left(d x^{\prime}\right)$ and thus we get the desired assertion.

Case 2. $p_{k}$ is even and there is only one odd number $p$ in $S(a)$. Let $p \neq$ $p_{1}, p_{k-1}$. We may assume $2 p \leq p_{k}$. Otherwise, we consider $\tilde{S}(a)$ and $p_{k}-p$. Write $\operatorname{gcd}\left(p_{1}, p_{2}, \ldots, p_{k-1}\right)=d$. So with $S^{\prime}(a)=\left\{0, p_{1} / d, \ldots, p_{k-1} / d\right\}$ we obtain for some $0 \leq x^{\prime}<p_{k-1} / d$ the relation (3.20). Having (3.20), we choose $l^{\prime}$ such that

$$
p_{k}-p+l d \leq d l^{\prime}+l d<p_{k}-p+(l+1) d, \quad \forall l=0, \ldots, p / d-1 .
$$

Next we apply Lemma 3.4 to get $y+l d=d l^{\prime}+l d-p_{k}+p \in \mathcal{B}\left(d x^{\prime}\right)$. Now it is clear that

$$
0 \leq y+d l<p, \quad \forall l=0, \ldots, p / d-1 .
$$

Moreover, $y-s p_{k} \equiv 0(\bmod d)$ if and only if $s+1=0(\bmod d)$. The same approach as in Case 1 (with $p$ instead of $p_{1}$ ) yields

$$
\{0, \ldots, p\} \cup\left\{l d: 0 \leq l d<p_{k}\right\} \subseteq \mathcal{B}\left(d x^{\prime}\right) .
$$

We know now how to get (3.1) from this relation.

To show (3.1) for $p=p_{1}$ or $p_{k-1}$, we should modify the above proof. Without loss of generality we may assume $p=p_{1}$ and $2 p_{1}>p_{k}$. So the $p_{j}, j=2, \ldots, k$, are even. Denote $\operatorname{gcd}\left(p_{1}, p_{2}, \ldots, p_{k-2}, p_{k}\right)=d_{1}$. With $S^{\prime}(a)=\left\{0, p_{1} / d_{1}, \ldots, p_{k-2} / d_{1}\right.$, $\left.p_{k} / d_{1}\right\}$ we obtain (3.20). To verify (3.1), let $q=p_{k}-p_{1}, q^{\prime}=p_{k-1} / 2$. So $\operatorname{gcd}\left(d_{1}, q^{\prime}\right)=1$. We already know that $l d_{1}+d_{1} \in \mathcal{B}\left(d_{1} x^{\prime}\right)$ for all $l=0,1, \ldots, q / d_{1}-1$. Now, since $q<p_{1}$, it follows from Lemma 3.1 that $l d_{1}+d_{1}+p_{1} \in \mathcal{B}\left(d_{1} x^{\prime}\right)$ while Lemma 3.4 with $p_{l_{1}}=p_{1}$ and $p_{l_{2}}=p_{k}$ implies $l d_{1}+d_{1}+p_{1}-\alpha q \in \mathcal{B}\left(d_{1} x^{\prime}\right)$ for $\alpha$ such that $q^{\prime} \leq l d_{1}+d_{1}+p_{1}-\alpha q<q^{\prime}+q$. Lemma 3.2 with $p_{k-1}$ leads to $l d_{1}+d_{1}+\left(p_{1}-q^{\prime}\right)-\alpha q \in \mathcal{B}\left(d_{1} x^{\prime}\right)$ and $0 \leq l d_{1}+d_{1}+\left(p_{1}-q^{\prime}\right)-\alpha q<q$. Now, let $y=d_{1}+\left(p_{1}-q^{\prime}\right)-\alpha q$. Then $y \in \mathcal{B}\left(d_{1} x^{\prime}\right)$ and $0<y<q$. Recursively, we obtain from the above procedure that $0 \leq y+l d_{1}+l_{s} d_{1}-s q^{\prime}<q$ and $y+l d_{1}+l_{s} d_{1}-s q^{\prime} \in \mathcal{B}\left(d_{1} x^{\prime}\right)$ for $s=0,1, \ldots, d_{1}-1$ and $l=0, \ldots, q / d_{1}-1$. Moreover, $y+l d_{1}+l_{s} d_{1}-s q^{\prime}=0$ if and only if $s=d_{1}-1$. Therefore, since $\operatorname{gcd}\left(d_{1}, q^{\prime}\right)=1$, these numbers are all different and are contained in $\{0, \ldots, q-1\}$. We conclude that

$$
\{v: 0 \leq v<q\} \subseteq \mathcal{B}\left(d_{1} x^{\prime}\right) .
$$

Again applying Lemma 3.1 with $p_{1}$, we obtain from (3.20) and the last relation that

$$
\{0,1, \ldots, q-1\} \cup\left\{p_{1}, \ldots, p_{k}-1\right\} \cup\left\{l d_{1}: \quad 0 \leq l d_{1}<p_{k}\right\} \subseteq \mathcal{B}\left(d_{1} x^{\prime}\right) .
$$

Let $p_{1} \leq v<p_{k}$. It follows from Lemma 3.4 with $p_{l_{1}}=p_{1}$ and $p_{l_{2}}=p_{k}$ that $v-\alpha q \in \mathcal{B}\left(d_{1} x^{\prime}\right)$ whenever $v-\alpha q \in \mathbb{Z}_{p_{k}}$. Combining this with (3.21) leads to (3.1).

Case 3. $p_{k}$ is odd. $S(a) \backslash\left\{p_{k}\right\}$ does not have property P. Thus, $S(a)$ contains an odd $p \neq p_{k}$ and an even $q \neq 0$. It is easy to see that $p_{1}, \ldots, p_{k-2}$ are even and $p_{k-1}$ is odd. Suppose $p_{k} \geq 2 p_{k-2}$, so $2 p_{j}<p_{k}$ for $j=1,2, \ldots, k-2$. Clearly $S(a) \backslash\left\{p_{2}\right\}=\left\{0, p_{1}, p_{3}, \ldots, p_{k}\right\}$ has property P. Denote $\operatorname{gcd}\left(p_{1}, p_{3}, \ldots, p_{k}\right)=d_{1}$ and $S^{\prime}(a)=\left\{0, p_{1} / d_{1}, p_{3} / d_{1}, \ldots, p_{k} / d_{1}\right\}$. We obtain (3.20) with some $0 \leq x^{\prime}<p_{k}$, i.e.,

$$
\left\{l d_{1}: 0 \leq l d_{1}<p_{k}\right\} \subseteq \mathcal{B}\left(d_{1} x^{\prime}\right) .
$$


Let $l^{\prime}$ satisfy

$$
p_{k}-p_{2}+l d_{1} \leq l^{\prime} d_{1}+l d_{1}<p_{k}-p_{2}+(l+1) d_{1} \quad \forall l=0, \ldots, p_{1} / d_{1}-1 .
$$

Clearly, for each such $l$ it holds that

$$
\left(2^{m}-1\right)\left(l^{\prime} d_{1}+l d_{1}-p_{2}\right)+k_{\eta}<\left(2^{m}-1\right)\left(p_{k}-p_{2}\right) .
$$

Hence, by Lemma 3.4 we conclude that

$$
l d_{1} \leq l^{\prime} d_{1}+l d_{1}-\left(p_{k}-p_{2}\right)<(l+1) d_{1} \text { and } l^{\prime} d_{1}+l d_{1}-\left(p_{k}-p_{2}\right) \in \mathcal{B}\left(d_{1} x^{\prime}\right) .
$$

Write $y=l^{\prime} d_{1}-\left(p_{k}-p_{2}\right)$. We obtain $y+s p_{2} \equiv 0\left(\bmod d_{1}\right)$ if and only if $s+1 \equiv 0$ $\left(\bmod d_{1}\right)$. We know that $p_{1}=2^{\mu} g$, where $g$ is odd. Also, $d_{1} \neq 1$ implies $g \neq 1$. For each $y+l d_{1}$ we choose $\epsilon_{i, j}=0$ for $j>1$ to obtain

$$
\begin{aligned}
2^{m}\left(y+l d_{1}\right)+k_{\eta}-\sum_{i=0}^{m-1} \sum_{j=1}^{k} \epsilon_{i, j} 2^{i} p_{j} & =2^{m}\left(y+l d_{1}\right)+k_{\eta}-\sum_{i=0}^{m-1} \epsilon_{i, 1} 2^{i} p_{1} \\
& =y+l d_{1}+\left(2^{m}-1\right)\left(y+l d_{1}\right)+k_{\eta}-\gamma p_{1} \\
& =y+l d_{1}+\left(2^{m}-1\right)\left(y+l d_{1}\right)+k_{\eta}-\gamma 2^{\mu} g,
\end{aligned}
$$

where $\gamma$ can be any integer between 0 and $2^{m}-1$. By the definition of $\mathcal{B}\left(d_{1} x^{\prime}\right)$ we conclude that $y+l d_{1}+\left(2^{m}-1\right)\left(y+l d_{1}\right)+k_{\eta}-\gamma 2^{\mu} g \in \mathcal{B}(x)$ whenever $y+l d_{1}$ $+\left(2^{m}-1\right)\left(y+l d_{1}\right)+k_{\eta}-\gamma 2^{\mu} g \in \mathbb{Z}_{p_{k}}$. Thus, we can choose $\gamma$ to obtain

$$
y+l d_{1}+\left(2^{m}-1\right)\left(y+l d_{1}\right)+k_{\eta}-\gamma 2^{\mu} g=y+l d_{1}+r p_{1}+\beta g
$$

with some $r$ and $0 \leq \beta \leq 2^{\mu}-1$ such that $p_{k}-p_{2} \leq y+l d_{1}+r p_{1}+\beta g<p_{k}$. It follows from Lemma 3.4 that $0 \leq y+l d_{1}+r p_{1}+\beta g-\left(p_{k}-p_{2}\right)<p_{2}$ is contained in $\mathcal{B}\left(d_{1} x^{\prime}\right)$. Furthermore, with $p_{l_{1}}=p_{1}$ and $p_{l_{2}}=p_{k}$ we get by Lemma 3.2 for some $\alpha \geq 0$ that

$$
0 \leq y+l d_{1}+r p_{1}+\beta g-\left(p_{k}-p_{2}\right)-\alpha g<p_{1}
$$

and

$$
y+l d_{1}+r p_{1}+\beta g-\left(p_{k}-p_{2}\right)-\alpha g \in \mathcal{B}\left(d_{1} x^{\prime}\right) .
$$

We may write with some $\alpha_{l, 1}$

$$
y+l d_{1}+r p_{1}+\beta g-\left(p_{k}-p_{2}\right)-\alpha g=y+l d_{1}+\alpha_{l, 1} d_{1}-\left(p_{k}-p_{2}\right) .
$$

Clearly, these new numbers are different from $y+l d_{1}$. Therefore with $\alpha_{l, 0}=0$ we conclude recursively for $s=0,1, \ldots, d_{1}-1, l=0, \ldots, p_{1} / d_{1}-1$ that

$0 \leq y+l d_{1}+\alpha_{l, s} d_{1}-s\left(p_{k}-p_{2}\right)<p_{1} \quad$ and $\quad y+l d_{1}+\alpha_{l, s} d_{1}-s\left(p_{k}-p_{2}\right) \in \mathcal{B}\left(d_{1} x^{\prime}\right)$.

The choice of $y$ ensures that these numbers are all different. Hence, together with (3.20) we get

$$
\left\{0,1, \ldots, p_{1}\right\} \cup\left\{l d_{1}: 0 \leq l d_{1}<p_{k}\right\} \subseteq \mathcal{B}\left(d_{1} x^{\prime}\right) .
$$

To finish our proof for the case $p_{k} \geq 2 p_{k-2}$, we have to verify $\left\{p_{1}+1, \ldots, p_{k}-1\right\} \subseteq$ $\mathcal{B}\left(d_{1} x^{\prime}\right)$. For this goal we assume first $k_{\eta} \neq 2^{m}-1$. Thus, $k_{\eta}$ is even. We apply (i) of Lemma 3.3 with $p_{l_{1}}=p_{k}$ and $p_{j^{\prime}}=p_{k-1}$ for each odd number from $\left\{0, \ldots, p_{1}\right\}$ to obtain

$$
\left\{x: 0 \leq x \leq p_{1}+p_{k}-p_{k-1}, \quad x \text { is odd }\right\} \cap \mathbb{Z}_{p_{k}} \subseteq \mathcal{B}\left(d_{1} x^{\prime}\right) .
$$

Recursively, we conclude

$$
\left\{x: 0 \leq x<p_{k}, \quad x \text { is odd }\right\} \subseteq \mathcal{B}\left(d_{1} x^{\prime}\right) .
$$


Next, we use Lemma 3.1 with $p_{k-1}$ for each number from $\left\{1, \ldots, p_{1}\right\}$ to get

$$
\left\{x: p_{k-1}<x \leq \min \left\{p_{k-1}+p_{1}, p_{k}-1\right\}\right\} \subseteq \mathcal{B}\left(d_{1} x^{\prime}\right) .
$$

Now for each even number $x$ between $p_{k-1}$ and $\min \left\{p_{k-1}+p_{1}, p_{k}-1\right\}$ we apply (ii) of Lemma 3.3 with $p_{l_{1}}=p_{k}$ and $p_{j^{\prime}}=p_{1}$. So, recursively, we obtain

$$
\left\{x: p_{1} \leq x \leq p_{k-1}, \quad x \text { is even }\right\} \subseteq \mathcal{B}\left(d_{1} x^{\prime}\right) .
$$

Combining this with (3.22) and (3.23), we get

$$
\left\{0, . ., p_{k-1}\right\} \subseteq \mathcal{B}\left(d_{1} x^{\prime}\right) .
$$

The desired assertion follows from Lemma 3.1 with $p_{k-1}$ and the last relation. Similarly, we have (3.1) for $k_{\eta}=2^{m}-1$.

It remains to show (3.1) for $2 p_{k-2} \geq p_{k}$. Thus $2\left(p_{k}-p_{k-2}\right) \leq p_{k}$. The symmetric relation (3.2) tells us that in this case we need only to verify (3.1) for the set $S(a)$ with even $p_{1}$ and odd $p_{2}, \ldots, p_{k}$, which satisfies $2 p_{1} \leq p_{k}$. Clearly $\left\{0, p_{1}, \ldots, p_{k-1}\right\}$ has property P. Denote $\operatorname{gcd}\left(p_{1}, \ldots, p_{k-1}\right)=d_{2}$. We have as before

$$
\left\{l d_{2}: 0 \leq l d_{2}<p_{k}\right\} \subseteq \mathcal{B}\left(d_{2} x^{\prime}\right) .
$$

Next, for $l=0, \ldots, p_{1} / d_{2}-1$ let $l^{\prime}$ be such that

$$
p_{k}-p_{1}+l d_{2} \leq l^{\prime} d_{2}+l d_{2}<p_{k}-p_{1}+(l+1) d_{2} .
$$

Write $y=l^{\prime} d_{2}-\left(p_{k}-p_{1}\right)$. Lemma 3.4 tells us that $y+l d_{2} \in \mathcal{B}\left(d_{2} x^{\prime}\right)$ for $l=0, \ldots$, $p_{1} / d_{2}-1$. We now know how to use Lemmas 3.1, 3.2 and 3.4 from these numbers to obtain, for $s=0, \ldots, d_{2}-1$ and $l=0, \ldots, p_{1} / d_{2}-1$,

$y+l d_{2}+\alpha_{s, l} d_{2}-s\left(p_{k}-p_{1}\right)<p_{1} \quad$ and $\quad y+l d_{2}+\alpha_{s, l} d_{2}-s\left(p_{k}-p_{1}\right) \in \mathcal{B}\left(d_{2} x^{\prime}\right)$, which in turn implies $\left\{0, \ldots, p_{1}-1\right\} \subseteq \mathcal{B}\left(d_{2} x^{\prime}\right)$. Hence,

$$
\left\{0, \ldots, p_{1}\right\} \cup\left\{l d_{2}: 0 \leq l d_{2}<p_{k}\right\} \subseteq \mathcal{B}\left(d_{2} x^{\prime}\right) .
$$

The assertion follows from Lemmas 3.1, 3.3 and this relation.

Case 4. $p_{k}$ is odd. $S(a) \backslash\left\{p_{k}\right\}$ has property $\mathrm{P}$ and $S(a) \backslash\left\{p_{k}\right\}$ contains an odd $p$ and an even $q \neq 0$. Denote $\operatorname{gcd}\left(p_{1}, \ldots, p_{k-1}\right)=d$. Thus, we obtain (3.20), i.e.,

$$
\left\{l d: 0 \leq l d<p_{k}\right\} \subseteq \mathcal{B}\left(d x^{\prime}\right) .
$$

For $q \leq p_{k}-q$ the proof is the same as in the last situation of Case 3. We omit the details here.

Let $q \geq p_{k}-q$. We may assume that $q$ is minimal among all nonzero even numbers in $S(a)$ and that $p$ satisfies $2 p<p_{k}$. Otherwise we consider $\tilde{S}(a)$, for which we have already the desired assertion. For the same reason we can also assume $p+q \leq p_{k}$. To this end, first let $2 p \geq q$. Denote $q^{\prime}=q / 2$. Furthermore, let $q \leq l^{\prime} d+l d<p_{k}$ for some $l^{\prime}$ and $l=0, \ldots, q^{\prime} / d-1$. By Lemma 3.4 we get $2 q-p_{k} \leq l^{\prime} d+l d-\left(p_{k}-q\right)<q$. We may use Lemma 3.2 to subtract $q^{\prime}$ from $l^{\prime} d+l d-\left(p_{k}-q\right)$ such that $0 \leq l^{\prime} d+l d-\left(p_{k}-q\right)-\alpha_{l} q^{\prime}<q^{\prime}$. Now write $y_{l}=l^{\prime} d+l d-\left(p_{k}-q\right)-\alpha_{l} q^{\prime}$. Thus,

$$
y_{l} \in \mathcal{B}\left(d x^{\prime}\right) \quad \text { and } \quad y_{l} \neq 0, \quad \forall l=0, \ldots, q^{\prime} / d-1 .
$$

Furthermore, they are all different and $y_{l}-s p_{k} \equiv 0(\bmod d)$ if and only if $s+1 \equiv 0$ $(\bmod d)$. Now we obtain recursively by applying Lemma 3.1 with $p$, Lemma 3.4 with $q$ and $p_{k}$ and finally Lemma 3.2 with $q$ that

$$
y_{l}+\alpha_{l, s} d+s\left(p_{k}-q\right) \in \mathcal{B}\left(d x^{\prime}\right) \quad \text { and } \quad 0 \leq y_{l}+\alpha_{l, s} d+s\left(p_{k}-q\right)<q^{\prime}
$$


for $l=0, \ldots, q^{\prime} / d-1$ and $s=0, \ldots, d-1$. Hence, together with (3.20) we conclude

$$
\left\{0, \ldots, q^{\prime}\right\} \cup\left\{l d: 0 \leq l d<p_{k}\right\} \subseteq \mathcal{B}\left(d x^{\prime}\right) .
$$

We verify that $q^{\prime}$ in the above relation can be replaced by $p$. If $q^{\prime} \geq p$, we have nothing more to do. If $q^{\prime}<p$, we use Lemma 3.1 with $p$ to obtain

$$
\left\{0, \ldots, q^{\prime}\right\} \cup\left\{p, \ldots, p+q^{\prime}\right\} \subseteq \mathcal{B}\left(d x^{\prime}\right) .
$$

For each number of $\left\{p, \ldots, p+q^{\prime}-1\right\}$ we apply Lemma 3.2 with $q$ to obtain $\left\{p-q^{\prime}, \ldots, p-1\right\} \subseteq \mathcal{B}\left(d x^{\prime}\right)$. Thus,

$$
\left\{p-q^{\prime}, . ., p+q^{\prime}\right\} \subseteq \mathcal{B}\left(d x^{\prime}\right)
$$

Again using Lemma 3.2 with $q$ for each element from $\left\{p-q^{\prime}, \ldots, p+q^{\prime}-1\right\}$, we obtain $\left\{p-2 q^{\prime}, \ldots, p+q^{\prime}\right\} \subseteq \mathcal{B}\left(d x^{\prime}\right)$. Recursively, $\left\{0, \ldots, p+q^{\prime}\right\} \subseteq \mathcal{B}\left(d x^{\prime}\right)$. Thus,

$$
\{0, \ldots, p\} \cup\left\{l d: 0 \leq l d<p_{k}\right\} \subseteq \mathcal{B}\left(d x^{\prime}\right) .
$$

Since $p$ is odd, (3.1) follows from Lemma 3.1 with $p$ and the last relation.

It remains to verify (3.1) for $2 p \leq q$. We should modify the procedure for $2 p \geq q$ as follows: let $p_{k}-p \leq l^{\prime} d+l d<p_{k}$ for some $l^{\prime}$ and $l=0, \ldots, p / d-1$. By Lemma 3.4 with $p_{k}$ and $q$ we get $q-p \leq l^{\prime} d+l d-\left(p_{k}-q\right)<q$. Again using Lemma 3.4 with $p$ and $q$, we conclude that $0 \leq l^{\prime} d+l d-\left(p_{k}-p\right)<p$. Now write $y_{l}=l^{\prime} d+l d-\left(p_{k}-p\right)$. Thus,

$$
y_{l} \in \mathcal{B}\left(d x^{\prime}\right) \quad \text { and } \quad y_{l} \neq 0, \forall l=0, \ldots, p / d-1 .
$$

We obtain in the same way that

$$
y_{l}+\alpha_{l, s} p+s\left(p_{k}-p\right) \in \mathcal{B}\left(d x^{\prime}\right) \quad \text { and } \quad 0 \leq y_{l}+\alpha_{l, s} p+s\left(p_{k}-p\right)<p
$$

for $l=0 \ldots, p / d-1$ and $s=0, \ldots, d-1$. Hence, together with (3.20) we conclude (3.24). From (3.24) we know how to get (3.1).

\section{ACKNOWLEDGMENT}

The author is indebted to the referee for various helpful comments on his paper.

\section{REFERENCES}

[1] M. Bröker and X. Zhou, Characterization of continous, four-coefficient scaling functions via matrix spectral radius, SIAM J. Matrix Anal. Appl., 22 (2000), 242-257. MR2001h:65169

[2] A. S. Cavaretta, W. Dahmen, and C. A. Micchelli, Stationary Subdivision, Mem. Amer. Math. Soc., 453 (1991). MR92h:65017

[3] G. M. Chaikin, An algorithm for high speed curve generation, Comp. Graphics and Image. Proc., 3 (1974), 346-349.

[4] D. Colella and C. Heil, Characterizations of scaling functions: continuous solutions, SIAM J. Matrix Anal. Appl., 15 (1994), 496-518. MR95f:26004

[5] I. Daubechies and J. C. Lagarias, Two-scale difference equations I. Existence and global regularity of solutions, SIAM J. Math. Anal., 22 (1991), 1388-1410. MR92d:39001

[6] D. E. Gonsor, Subdivision algorithms with nonnegative masks generally converge, Adv. Comp. Math., 1 (1993), 215-221. MR.94e:65023

[7] R. -Q. Jia, Subdivision schemes in $L_{p}$ spaces, Advances in Comp. Math, 3 (1995), 309-341. MR96d:65028

[8] R. -Q. Jia and D. -X. Zhou, Convergence of subdivision schemes associated with nonnegative masks, SIAM J. Matrix Anal. Appl., 21 (1999), 418-430. MR2001a:42041

[9] A. A. Melkman, Subdivision schemes with non-negative masks always converge - unless they obviously cannot?, Ann. Numer. Math., 4 (1997), 451-460. MR97i:41014

[10] C. A. Micchelli and H. Prautzsch, Uniform refinement of curves, Linear Algebra Appl., 114/115 (1989), 841-870. MR.90k:65088 
[11] G. C. Rota and G. Strang, A note on the joint spectral radius, Indag. Math., 22 (1960), 379-381. MF 26:5434

[12] G. de Rham, Sur une courbe plane, J. Mathem. pures et appl., 39 (1956), 25-42. MR 19:842e

[13] J. N. Tsitsiklis and V. D. Blondel, The Lyapunov exponent and joint spectral radius of pairs of matrices are hard, when not impossible, to compute and to approximate, Mathematics of Control, Signals and Systems, 10 (1997), 31-41. MR99h:65238a

[14] Y. Wang, Two-scale dilation equations and the cascade algorithm, Random and Computational Dynamics, 3 (1995), 289-309. MR 96m:42060

[15] Y. Wang, Subdivision schemes and refinement equations with nonnegative masks, J. Approx. Th., 113 (2001), 207-220. MR2002i:42054

[16] D.-Z. Zhou, The p-norm joint spectral radius for even integers, Methods and Applications of Analysis, 5 (1) (1998), 39-54. MF99e:42054

Department of Mathematics, China Jiliang University, Hangzhou, China; Institute of Mathematics, University of Duisburg-Essen, D-47057 Duisburg, Germany

E-mail address: zhou@math.uni-duisburg.de 